San Jose State University

SJSU ScholarWorks

Master's Projects

Master's Theses and Graduate Research

Spring 2016

\title{
EFFICIENT PAIR-WISE SIMILARITY COMPUTATION USING APACHE SPARK
}

Parineetha Gandhi Tirumali

San Jose State University

Follow this and additional works at: https://scholarworks.sjsu.edu/etd_projects

Part of the Databases and Information Systems Commons

\section{Recommended Citation}

Tirumali, Parineetha Gandhi, "EFFICIENT PAIR-WISE SIMILARITY COMPUTATION USING APACHE SPARK" (2016). Master's Projects. 479.

DOI: https://doi.org/10.31979/etd.sh8a-3gyv

https://scholarworks.sjsu.edu/etd_projects/479

This Master's Project is brought to you for free and open access by the Master's Theses and Graduate Research at SJSU ScholarWorks. It has been accepted for inclusion in Master's Projects by an authorized administrator of SJSU ScholarWorks. For more information, please contact scholarworks@sjsu.edu. 


\title{
EFFICIENT PAIR-WISE SIMILARITY COMPUTATION USING APACHE
} SPARK

\author{
A Project \\ Presented to \\ The Faculty of the Department of Computer Science \\ San Jose State University
}

\author{
In Partial Fulfillment \\ of the Requirements for the Degree \\ Master of Science
}

By

Parineetha Gandhi Tirumali

May 2016 
(C)2016

Parineetha Gandhi Tirumali

ALL RIGHTS RESERVED 
The Designated Thesis Committee Approves the Thesis Titled

EFFICIENT PAIR-WISE SIMILARITY COMPUTATION USING APACHE SPARK

\author{
by \\ Parineetha Gandhi Tirumali \\ APPROVED FOR THE DEPARTMENT OF COMPUTER SCIENCE \\ SAN JOSE STATE UNIVERSITY \\ May 2016 \\ Dr. Tran Duc Thanh Department of Computer Science. \\ Dr. Thomas Austin Department of Computer Science. \\ Mr. Subrahmanyam Bolla Manager, VMWare.
}




\section{ABSTRACT \\ EFFICIENT PAIR-WISE SIMILARITY COMPUTATION USING APACHE SPARK \\ by Parineetha Gandhi Tirumali}

Entity matching is the process of identifying different manifestations of the same real world entity. These entities can be referred to as objects(string) or data instances. These entities are in turn split over several databases or clusters based on the signatures of the entities. When entity matching algorithms are performed on these databases or clusters, there is a high possibility that a particular entity pair is compared more than once. The number of comparison for any two entities depend on the number of common signatures or keys they possess. This effects the performance of any entity matching algorithm. This paper is the implementation of the algorithm written by Erhard Rahm et al. for performing redundancy free pair-wise similarity computation using MapReduce. As an improvisation to the existing implementation, this project aims to implement the algorithm in Apache Spark in standalone mode for sample of data and in cluster mode for large volume of data. 


\section{ACKNOWLEDGEMENTS}

I would like to thank the following people and many others who aren't named here.

I would like to express my gratitude to Professor Tran for his continuous support and guidance throughout the completion of this project.

I would like to thank my committee members Dr. Thomas Austin and Mr. Subrahmanyam Bolla for their contribution.

I would like to thank my parents, family and my husband for their cooperation. 


\section{Table of Contents}

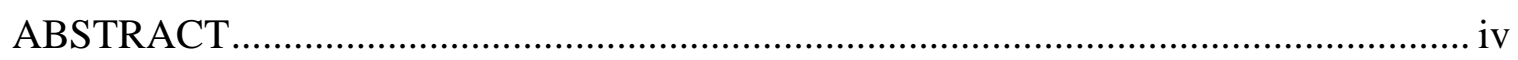

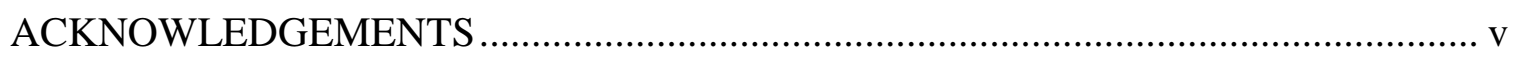

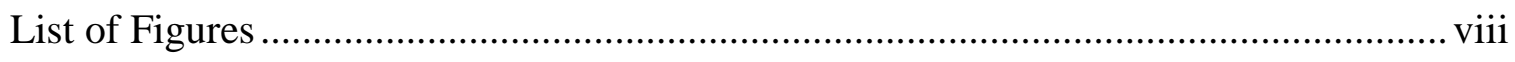

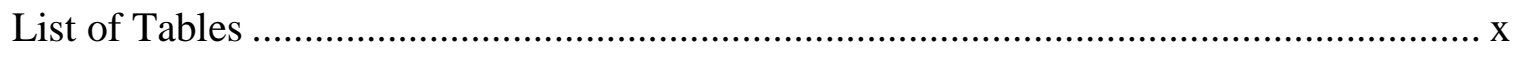

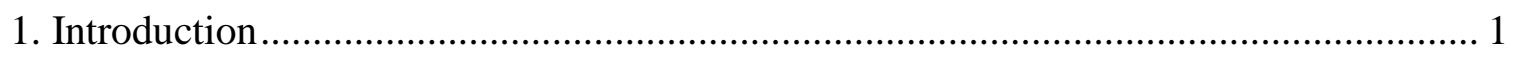

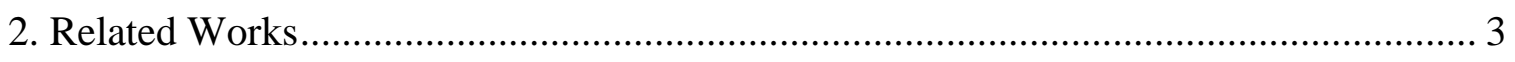

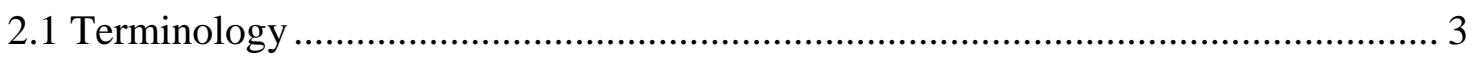

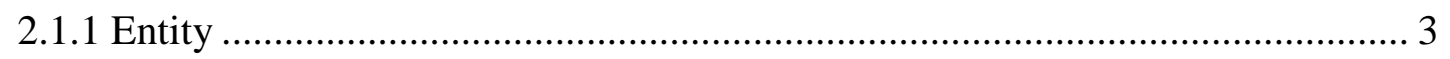

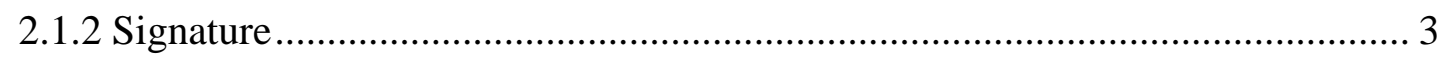

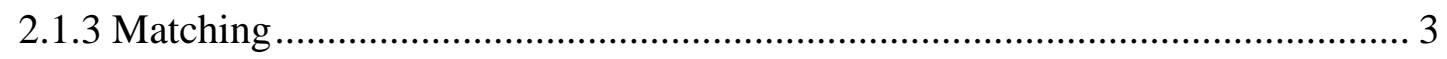

2.2 Pair-wise similarity computation(PSC) ............................................................. 3

2.3 Spark Implementation of Map Reduce ................................................................... 3

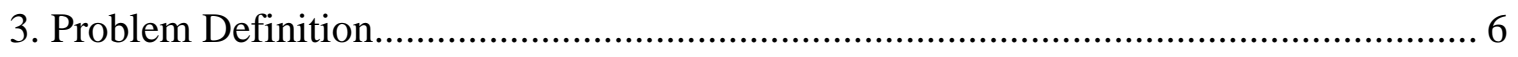

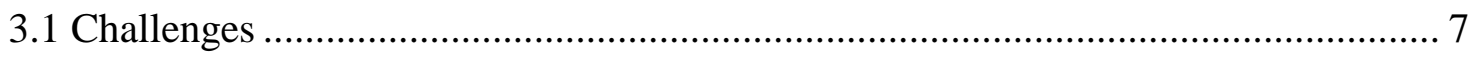

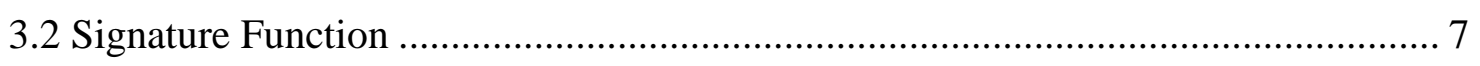

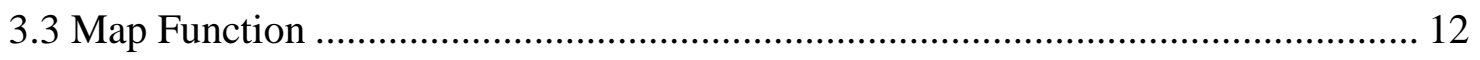

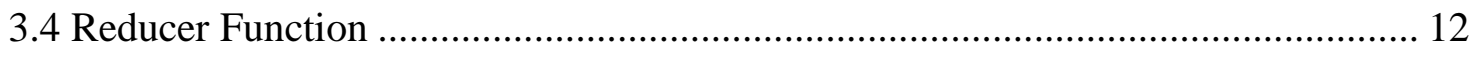

3.5 Real Time Example ..................................................................................... 14

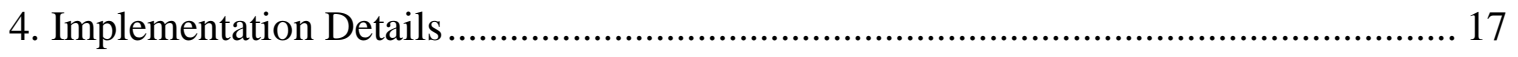

4.1 Baseline Implementation 1 ........................................................................... 17

4.2 Baseline Implementation 2 ......................................................................... 21

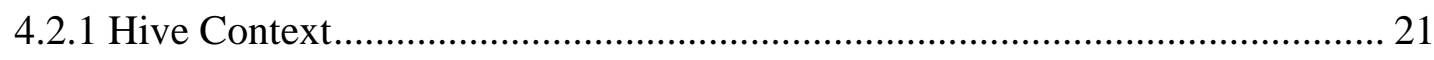

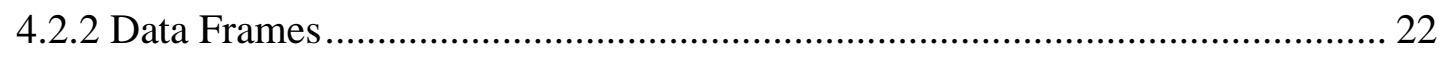




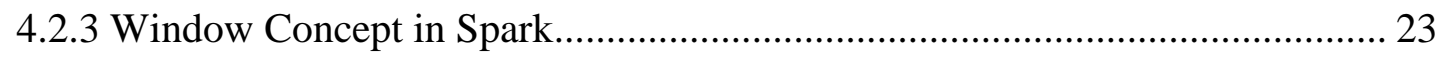

5. Performance Evaluation .................................................................................. 26

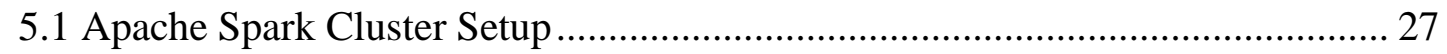

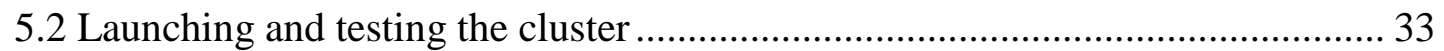

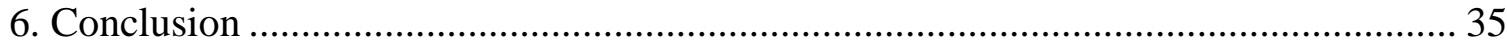

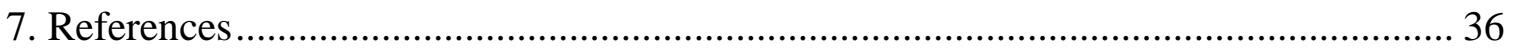




\section{List of Figures}

Figure 1: Referencing same paper object multiple times.......................................... 1

Figure 2: Multiple entries for the same product.................................................... 1

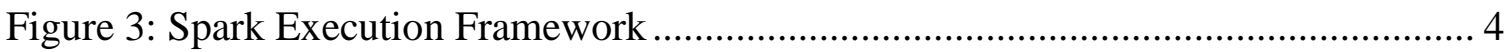

Figure 4: Python Spark Data Flow Architecture............................................................ 5

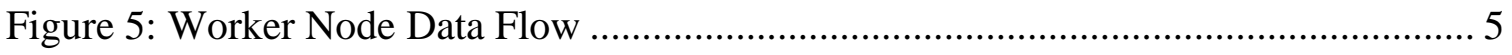

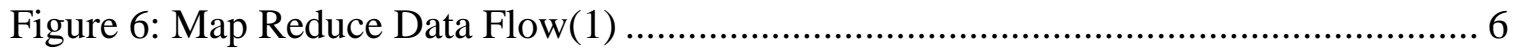

Figure 7: Difference between Map Reduce and Spark ................................................ 7

Figure 8: Example of Pair-wise similarity computation .......................................... 8

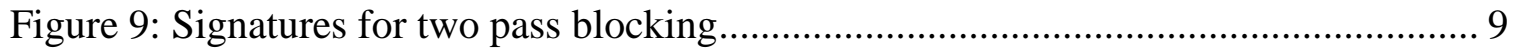

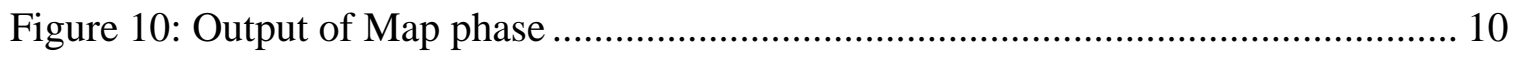

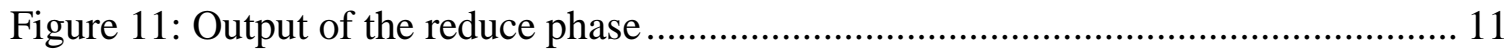

Figure 12: Map Reduce phase after improvising the algorithm ................................... 13

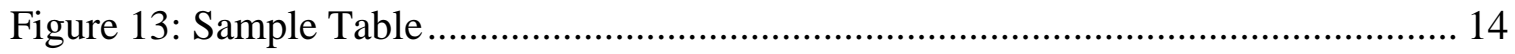

Figure 14: Key value pairs generated by Map ...................................................... 14

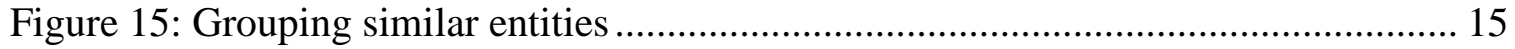

Figure 16: Sorting the keys associated with each entity ......................................... 15

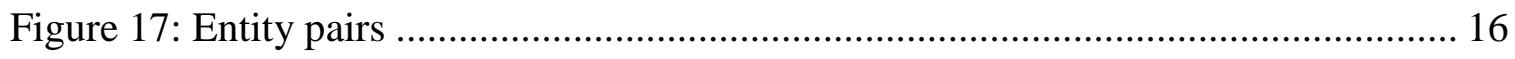

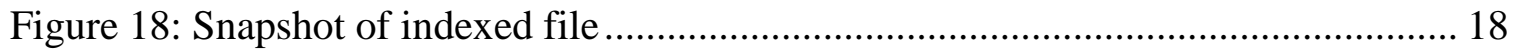

Figure 19: Code snippet to generate key value pairs from map .................................. 18

Figure 20: Snapshot of indexed file contents.......................................................... 19

Figure 21: Snapshot of Entity, SortedSignatures ....................................................... 19

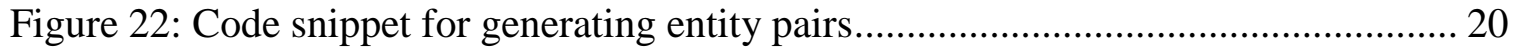

Figure 23: Snapshot of Key value pairs in the form of key and pairs of entities............. 20

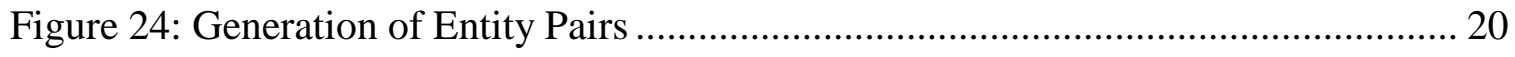

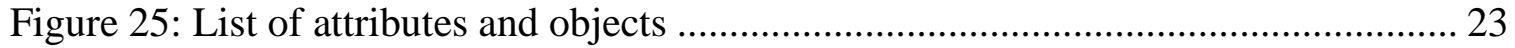

Figure 26: List of attributes, objects and previous values ......................................... 24

Figure 27: List of attributes and object, previous pairs .......................................... 24

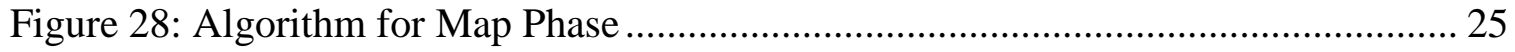




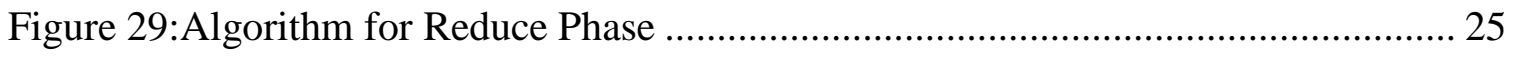

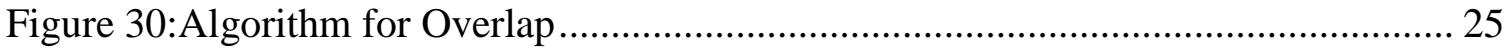

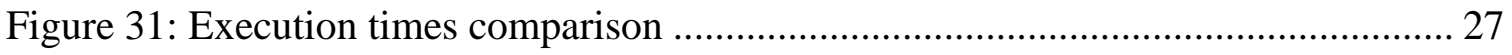

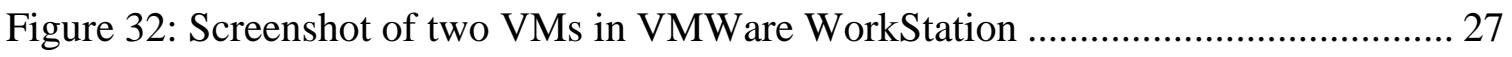

Figure 33: Screenshot showing Java version on VM................................................. 28

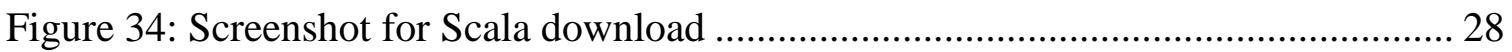

Figure 35: Screenshot showing Scala version on VM ........................................... 28

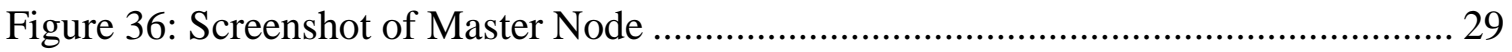

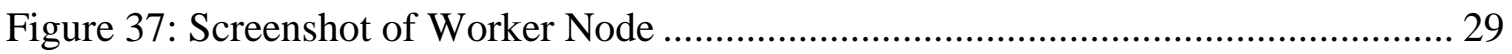

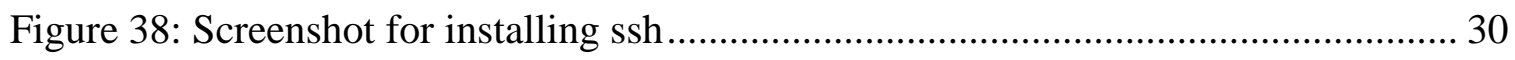

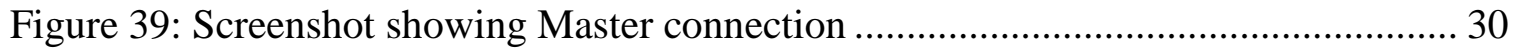

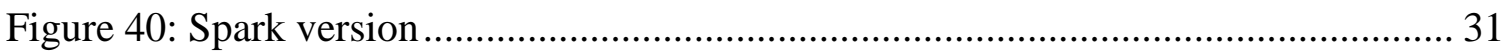

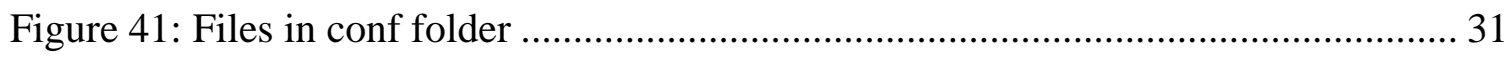

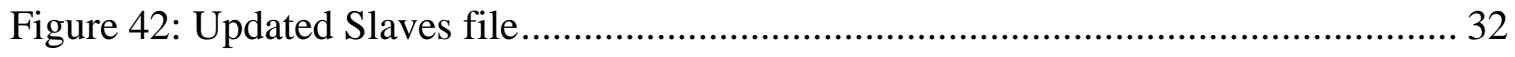

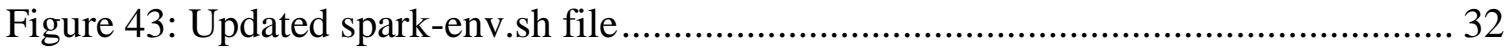

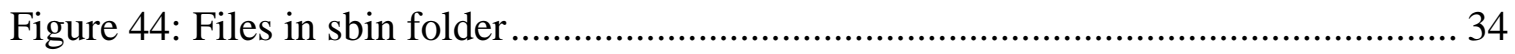




\section{List of Tables}

Table 1: Execution time with and without redundant pairs ......................................... 26 


\section{Introduction}

Entity matching is the process of identifying different manifestations of the same real world entity. An entity can be an object, data instance or a record. Examples of manifestations and objects include: different ways of addressing( names, email addresses) the same person; web pages with different descriptions of the same business; different photos of the same object and so on. The matching is performed by implementing several techniques like numerical matching approach, rule-based matching approach and workflow-based matching approach[1].

Some of the examples of entity matching are

\section{Example 1:}

\begin{tabular}{|c|c|c|c|}
\hline Title & Author & Venue & Year \\
\hline The merge/purge problem for large databases & $\begin{array}{l}\text { M.A. Hernandez, S.J. } \\
\text { Stolfo }\end{array}$ & Proceedings of the ACM SIGMOD international conference & \\
\hline The Merge/Purge problem for Large Databases & $\begin{array}{l}\text { A.H. Mauricio, J.S. } \\
\text { Stolfo }\end{array}$ & $\begin{array}{l}\text { Proc. of the } 1995 \text { ACM SIGMOD conference on } \\
\text { management }\end{array}$ & 1995 \\
\hline $\begin{array}{l}\text { andez, SJ Stolfo The merge/purge problem for Large } \\
\text { Databases }\end{array}$ & M. Hern & $\begin{array}{l}\text { Proceedings of the } 1995 \text { ACM SIGMOD conference on } \\
\text { management }\end{array}$ & 1995 \\
\hline
\end{tabular}

Figure 1: Referencing same paper object multiple times

Example 2:

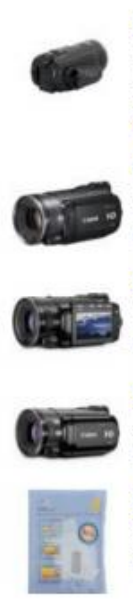

Canon VIXIA HF S10 Camcorder-1080p-8.59 MP - $10 \times$ optical zoom

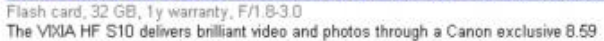
megapixel CMOS image sensor and the latest version of Canon's advanced image

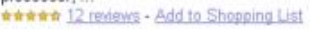

Canon ( VIXIA) HF S10 iVIS Dual Flash Memory Camcorder

Canon HF STOMS Dual Flash Memory CamcorderSPECCAL SALE PRiCE: $\$ 999$ Add to Shonging lit

Canon VIXIA HF S10

Dual Flash Memory High Definition Camcorder The Next Step Forward in HO Vided

Canon has a well-known and highly-regarded reputation for optical excellence, ....

Canon VIXIA HF S100 Flash Memory Camcorder

"Canon Video HF S100 instant Rebate Receme $\$ 200$ with your purchase of a new

Canon VXIA HF S100 Flash Memory Camcorder. (Price above includes $\$ 200 \ldots$

add to Shonging List

Canon Vixia Hf S10 Care \& Cleaning

Care \& Cleaning Digitar Camera/Camcorder Deluxe Cleaning Kt with LCD Screen

Guard Canon VIXIA HF S10 Camcorders Care \& Cleaning.

Add to Shopoing List

$\$ 975$ new

from 52 sellers -7

Compare prices

$\$ 899.00$ new

Made in Japan Online

$\$ 999.00$ new

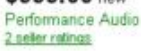

$\$ 899.95$ new

Avlingtoncamera com
5 aeler retinge

Figure 2: Multiple entries for the same product

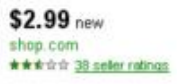


To say if two objects or two products or two people are same or not we need to compare them. To know if two entities are a match or non match, we need to first compare the pairs of entities. Due to the different manifestation of an entity and presence of multiple signatures or attributes for each entity, the entities will get compared more than once. The other reason for a particular pair of entity to be compared more than once is due to the presence of overlapping clusters. One of the processes for entity matching is blocking, this blocking is performed based on a blocking key. There is a possibility that an entity can have more than one blocking key, because of which the entities gets to share more than one cluster. So the comparison takes place more than once, due to which the efficiency deteriorates.

What motivates for Entity matching is linking census records, public health, web search, comparison shopping, portals integration from multiple sources, electronic marketplaces, integrating genomic data in medical genetics, monitoring events in the sky in the field of astrophysics.

The remaining part of this paper is organized as: section 2 gives a brief on related works done in pair wise similarity computation and its drawbacks. Section 3 describes about the problem definition and section 4 explains how this problem has been addressed.

Entities are distributed among the clusters or databases and when a comparison is performed on these clusters or databases, there is a high chance that the entities are compared redundantly. This reduces the efficiency of entity matching. One naive way of increasing the efficiency in terms of speed is using map reduce. But this does not solve the problem of redundant entity comparison completely. So this paper tries to solve the problem of redundant entity comparison using the concept of data frames and windows in Apache Spark.

The terms Entity and Object, and the terms Signature and Keys are one and the same throughout the paper. 


\section{Related Works}

Here after in this paper I use the following terminology to explain the concepts. This chapter gives a brief about these terminologies

\subsection{Terminology}

\subsubsection{Entity}

An entity in real world can be a person, product or any object which has attributes associated to it. An entity can also be referred to as an object, string or a document. Example: name, product.

\subsubsection{Signature}

A signature is associated with the entity, it can be a token, blocking key or set of terms. Example: category.substr(0,3) or manufacturer.

\subsubsection{Matching}

It is a process of comparing two entities and saying if they are a match or nonmatch.

Examples: Matching products for comparison shopping. Finding duplicate entries of customers in enterprise database

\subsection{Pair-wise similarity computation(PSC)}

Pair-wise similarity computations is an important concept in data related applications like entity resolution, clustering based on entities, etc. Groups of entities with same signature fall into one cluster and this is called clustering. During this process there is a high chance that entities getting duplicated in case of common signatures. How to deal with such scenario is the whole idea of implementing this paper.

\subsection{Spark Implementation of Map Reduce}

Apache Spark is an open source project found by UC Berkeley AMP Labs, the main motive of this project was to use in-memory, distributed data structure to speed up data processing over Hadoop. Map reduce concept was the early trial of making the 
process execution faster over distributed data structure, but introduction of spark made programs way more flexible and faster compared to map reduce alone.

In map reduce framework, few tasks are assigned to map and few to reducers. But, spark has a generic executor(JVM) depending on a situation executes map stages and reduces. JVM is core where all computation is executed, it is also an interface for other ecosystems like Hadoop. Consider we need to process 1TB of data on AWS, and the one worker node processes $1 \mathrm{~GB}$ of data in map stage the result is stored as 1 RDD.

Using Java or Scala will run the process directly on JVM. But for python the execution framework is different, it has several python or pyspark processes, generally one per task depending on the application. These processes are connected to JVM and data is shipped from JVM to python for processing.

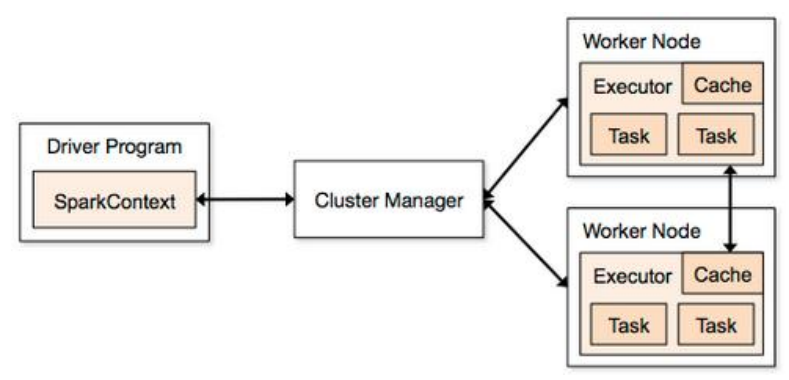

Figure 3: Spark Execution Framework

There can be several such worker nodes but there should be only one manager to provision or restart workers. This is called Cluster Manager. The object that connects and holds the cluster in spark is spark context. Spark's driver program directs the operations by initializing the spark context. Spark's actions and transformations are initialized in this spark context and when the program gets executed the worker nodes kick starts and process the data. Following figures show how the data flow when using python in Spark. 


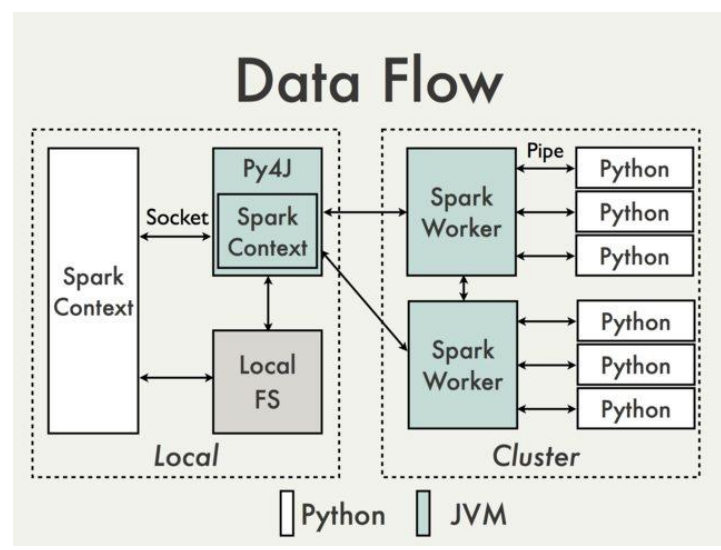

Figure 4: Python Spark Data Flow Architecture

Spark supports two interfaces of cluster management: yarn and standalone. Yarn is Hadoop's cluster manager which can be used with Hadoop map reduce and spark. Whereas a standalone interface has special spark process which takes care of starting the nodes that are failing.

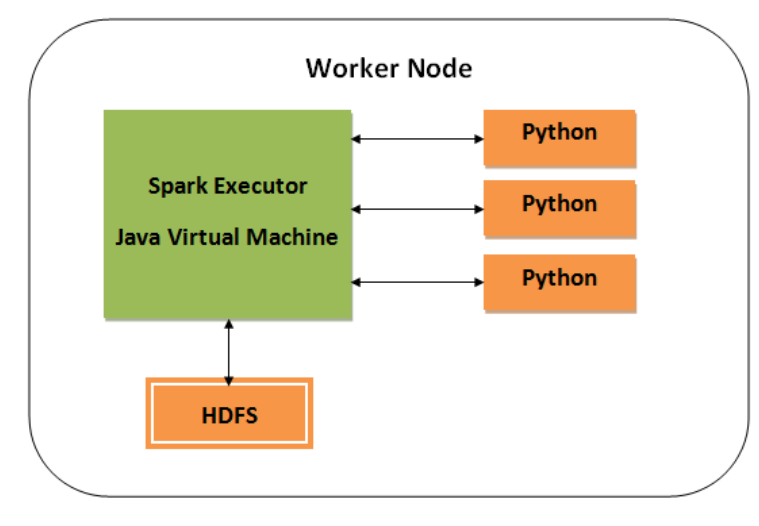

Figure 5: Worker Node Data Flow 


\section{Problem Definition}

Data quality play an important role for entity matching. Big data is massive representation of data and to find matching entities is very crucial and challenging task. This project mainly implements the concept of pair wise redundancy free comparison using Apache Spark. The basic approach for pair-wise similarity computation takes Cartesian product of the entity pairs. Cartesian product gives a complexity of $\mathrm{O}\left(\mathrm{n}^{2}\right)$ which is very high in terms of big data. This can be improvised by using Map Reduce concept to parallelize the computation which in turn speeds up the process but the quadratic complexity seems to be almost same even after using Map Reduce. So the paper referred modifies the algorithm for reduce phase. Following figure shows how the basic map reduce works.

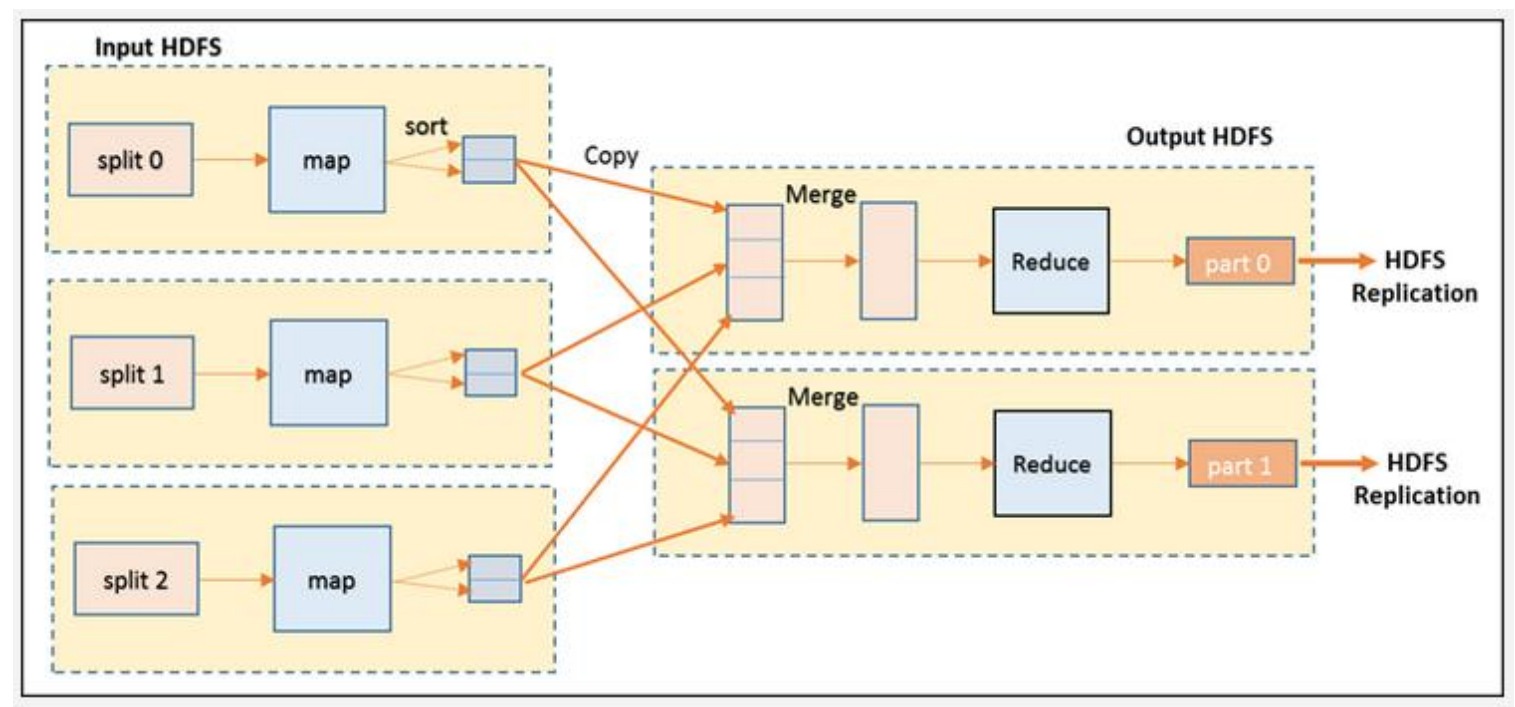

Figure 6: Map Reduce Data Flow(1)

Map Reduce alone sometimes is not so efficient when compared to Spark. Following are few differences between Map Reduce and Spark and we can clearly see that Spark out performs extraordinarily when compared to Map Reduce. 


\begin{tabular}{|c|c|c|}
\hline Criteria & Map Reduce & Spark \\
\hline Conciseness & Plain MR has a lot of boiler plate & Almost no boilerplate \\
\hline Performance & High latency & very fast compared to $M R$ \\
\hline Testability & Possible via libraries, but non trivial & Very much easy \\
\hline Iterative processing & Non trivial & straight forward \\
\hline Exploration of data & Not possible easily & $\begin{array}{l}\text { Spark shell allows quick and easy } \\
\text { data exploration }\end{array}$ \\
\hline SQL like interface & Via Hive & Build in as SparkSQL \\
\hline Fault Tolerance & $\begin{array}{l}\text { Inheranlty able to handle fault tolerance via } \\
\text { persisting the results of each of phases }\end{array}$ & $\begin{array}{l}\text { Exploits immutability of RDD to } \\
\text { enable fault tolerance }\end{array}$ \\
\hline Eco system & $\begin{array}{l}\text { lots of tools available but integration is not } \\
\text { quite seamless, requiring lot of effort for } \\
\text { their seamless integration }\end{array}$ & $\begin{array}{l}\text { Unifies lot of interfaces like SQL, } \\
\text { stream processing etc into single } \\
\text { abstraction of RDD }\end{array}$ \\
\hline $\begin{array}{l}\text { In memory } \\
\text { computations }\end{array}$ & not possible & possible \\
\hline
\end{tabular}

Figure 7: Difference between Map Reduce and Spark

\subsection{Challenges}

Matching entities while dealing with big data can be a tedious process and the quality of the data place a major role. When the data is so enormous in size it is obvious that it can be heterogeneous and there lies the challenging part. Heterogeneous data is unclean, unstructured and incomplete. With the growing data, applications and relationship between various sources of data, the need for matching is also growing. With this growth matching names with names is not as important as matching Amazon profiles with browsing history on Google and friends profile on Facebook. Larger datasets need efficient parallel techniques to process them.

\subsection{Signature Function}

The main problem in entity matching is that a particular entity pair comparison takes place many times, this leads to redundant pair comparison which reduces the efficiency of entity matching. The solution for elimination of redundant pair comparisons can be achieved by efficiently integrating with a parallel MR implementation.

Redundant comparison takes place when there are more than one common signatures between two entities. The basic map reduce can be improved by introducing the concept 
of clustering. The search space to match particular entity is reduced by grouping them with the entities of similar entities, and this group forms a cluster. Every cluster has the entities which are similar and the comparison takes place for pairs of entities present within the cluster.

For every entity in a group of entities $\mathbf{O}$, a sub group of attributes $\mathbf{S}$ are generated using the following signature function

$$
\sigma: 0 \rightarrow \mathcal{P}(S)
$$

This function takes group of entities and the attributes $\mathbf{S}$ as input and generate subset of attributes $\mathbf{s} \subseteq \mathbf{S}$ for each entity $\mathbf{o} \in \mathbf{O}$. The pair-wise similarity algorithm generates the similarity for all entity pairs that have minimum one common attribute.

$$
\{(01,02) \mid(01,02) \in O \text { X O } \Lambda \text { o1 } \neq \neq 02 \Lambda \sigma(01) \cap \sigma(02) \neq \emptyset\}
$$

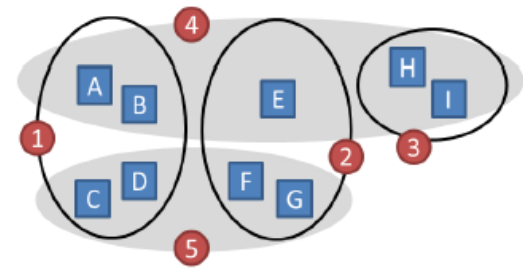

Figure 8: Example of Pair-wise similarity computation

In real time A, B, C.. can represents the product name, person name etc. 1, 2, 3.. can be price of a product, manufacturer or substring of title or category etc.

Consider A, B, C, D, E, F, G, H, I are few entities having 1, 2, 3, 4, 5 as keys or signatures. If these entities are not clustered there is a high possibility that these entities can be compared more than once. So the blocking algorithm is first performed to reduce the search space for matching. The figure shown above looks like it is the result of two pass blocking. Because of the presence of the more number of common signatures the entity pairs get compared once for each common signature. Generating signatures for the entities is part of the blocking phase. Blocking can be done based on one pass, or two 
pass or multi pass. The result obtained with multi pass is considered to be more accurate to find duplicates than single pass, because the entities which are not grouped in the first block gets grouped properly in the subsequent block phase. As the number of passes increases the size of the cluster gets smaller. The drawback of smaller cluster size is the similar entities pairs are missed. These missed entity pairs will never get compared with each other. The concept of blocking is beyond the scope of this project. Deciding on how many keys to generate and what keys to generate for entities is a difficult task, which depends on number of entities per cluster. For example, if the employee entities are clustered based on the address, there is a possibility that the same employees might be placed in different clusters if the address is slightly varying or missing.

After performing the two pass blocking on Figure 8 the resultant signature function generates signatures as shown in Figure 8

\begin{tabular}{|c|c|}
\hline Object & Signature \\
\hline A & $\{1,4\}$ \\
\hline B & $\{1,4\}$ \\
\hline C & $\{1,5\}$ \\
\hline D & $\{1,5\}$ \\
\hline E & $\{2,4\}$ \\
\hline F & $\{2,5\}$ \\
\hline G & $\{2,5\}$ \\
\hline H & $\{3,4\}$ \\
\hline I & $\{3,4\}$ \\
\hline
\end{tabular}

Figure 9: Signatures for two pass blocking

The first blocking phase generates three clusters with keys 1, 2, 3 and the second blocking phase generates clusters with 4, 5 keys. The map phase generates key value pairs for each entity. The output of the map phase is fed to the reducers through the partitioners. The partitioner performs some function over the keys, in this particular example the function is finding the modulo. The key value is divided by the number of keys and as per the result the partitioner send the key value pairs to the reducers. The signatures 1, 3, 5 are passed to one reducer and the signatures with 2 and 4 are passed to the other reducer. The output of the map phase is shown below. 


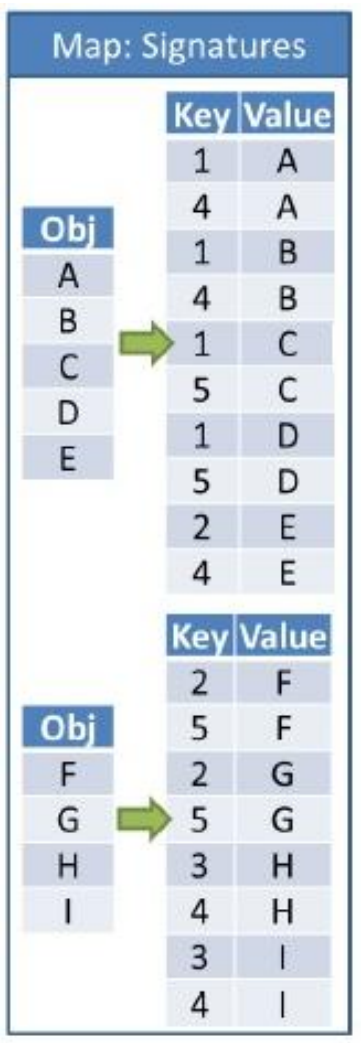

Figure 10: Output of Map phase

Pair-wise comparison takes place in reducer for each key. Due to the presence of the common signatures the entities are compared redundantly in the reduce phase. The entities that are compared redundantly are underlined in the figure shown below. This is due to the presence of overlapping cluster. So there is a need to change the processing of the reduce phase which can avoid redundant comparisons. The output of the reduce phase before changing the algorithm is shown in Figure 10. 


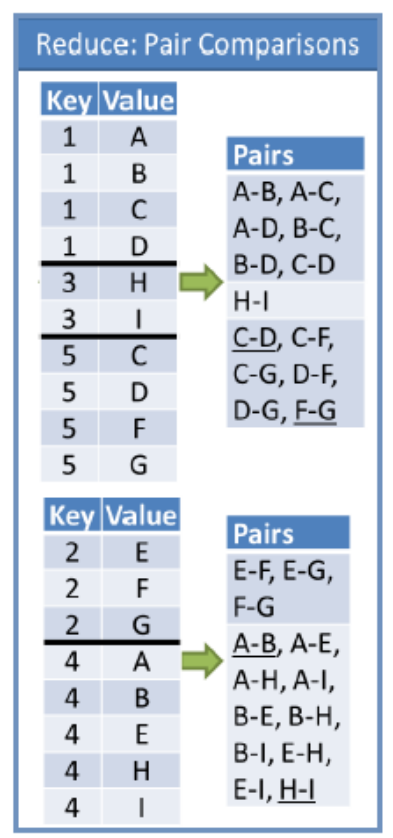

Figure 11: Output of the reduce phase

The main reason for the redundant comparison to take place is that one reducer is not aware of what entity pairs other reducers are processing. So to make the reducers smarter we need to implement a small function to calculate the minimum value among the list of common keys for any two entity pairs.

\section{$l \in \min (\sigma(01) \cap \sigma(02))$}

There can be various other approaches to solve this problem instead of just finding the smallest key among the list of common keys. Now the reducer that handles the signature 1 is responsible for comparing the entities o1 and 02 . No other reducer will compare these two entities again. How does this work? The reducer receives entities o1 and 02 as input and the partitioner. The reducers checks if there is any signature less than the current signature produced by the partitioner. If there exists a signature less than that then the reducer will not compare these two entities as it assumes that this pair is taken care by the other reducer. If there is no signature less than the one produced by the partitioner then the reducer takes that entity pair and compares. 


\subsection{Map Function}

The output of the map function is modified from just generating the key value pairs to generating the subgroup of keys smaller than the key received. Initially the map outputted the list of keys for a particular entity i.e.,

$$
\begin{gathered}
\sigma(\mathbf{o})=\left\{\mathbf{s}_{\mathbf{1}}, \mathrm{s}_{2}, \mathrm{~s}_{3}, . . \mathrm{s}_{\mathbf{n}}\right\} \\
\text { Let } \boldsymbol{\sigma}_{\mathrm{si}}(\mathbf{o})=\mathbf{s} \in \boldsymbol{\sigma}(\mathbf{0}) \mid \mathrm{s}<\mathrm{s}_{\mathbf{i}}
\end{gathered}
$$

After improvisation the map function now emits

$$
\left(\mathbf{s}_{\mathbf{i}},\left[\mathbf{o}, \boldsymbol{\sigma}_{\mathrm{si}}(\mathbf{o})\right]\right) \text { for every } \mathbf{1} \leq \mathbf{i} \leq \mathbf{n}
$$

\subsection{Reducer Function}

Reducer takes the above input and for present key $\mathrm{k}$ and the entity pair similar to the one shown above performs an extra step of checking if the two entities have disjoint key set. For a given pair $\left(\left[\mathbf{o}_{\mathbf{1}}, \boldsymbol{\sigma}_{\mathrm{k}}\left(\mathbf{o}_{1}\right)\right],\left[\mathbf{o}_{\mathbf{2}}, \boldsymbol{\sigma}_{\mathrm{k}}\left(\mathbf{o}_{2}\right)\right]\right)$ the reducer checks if

$$
\sigma_{k}\left(o_{1}\right) \cap \sigma_{k}\left(o_{2}\right)=\emptyset
$$

If they are disjoint the reducer makes the current key as the least common key and the two entities o1 and 02 are compared. If these two sets are not mutually exclusive then it means that there is/are smaller keys $\mathrm{k}^{\prime}$ is present for that pair of entity $\left(\mathrm{o}_{1}, \mathrm{o}_{2}\right)$. So in this scenario the key $\mathrm{k}$ is not considered. 


\begin{tabular}{|c|c|c|c|c|c|c|c|}
\hline \multicolumn{3}{|c|}{ Map: Signatures } & & \multicolumn{4}{|c|}{ Reduce: Pair Comparisons } \\
\hline \multirow{4}{*}{ Obj } & Key & Value & & Key & Value & \multirow{4}{*}{$\begin{array}{l}\text { Pairs } \\
\text { A-B, A-C, }\end{array}$} & \multirow{3}{*}{$\begin{array}{l}\text { Eliminated } \\
\text { Pairs }\end{array}$} \\
\hline & 1 & $A, \varnothing$ & & 1 & $A, \varnothing$ & & \\
\hline & 4 & $A,\{1\}$ & & 1 & $B, \varnothing$ & & \\
\hline & 1 & $B, \varnothing$ & & 1 & $C, \varnothing$ & & \multirow{3}{*}{ - } \\
\hline A & 4 & $B,\{1\}$ & & 1 & $D, \varnothing$ & \multirow{2}{*}{$\begin{array}{l}\text { A-D, B-C, } \\
\text { B-D, C-D }\end{array}$} & \\
\hline \multirow{2}{*}{$\begin{array}{l}\text { B } \\
\text { C }\end{array}$} & 1 & $C, \varnothing$ & & 3 & $\mathrm{H}, \varnothing$ & & \\
\hline & 5 & $C,\{1\}$ & 을 & 3 & $1, \varnothing$ & \multirow{3}{*}{$\begin{array}{l}\text { H-I } \\
\text { C-F, C-G, } \\
\text { D-F, D-G }\end{array}$} & \multirow{3}{*}{$\begin{array}{l}- \\
\text { C-D: }\{1\} \cap\{1\} \\
\text { F-G: }\{2] \cap\{2]\end{array}$} \\
\hline \multirow{2}{*}{$\begin{array}{l}\mathrm{D} \\
\mathrm{E}\end{array}$} & 1 & $D, \varnothing$ & 뭉 & 5 & $C,\{1\}$ & & \\
\hline & 5 & $D,\{1\}$ & $\frac{\varepsilon}{\lambda}$ & 5 & $D,\{1\}$ & & \\
\hline & 2 & $E, \varnothing$ & $\underline{\underline{\omega}}$ & 5 & $F,\{2\}$ & \multirow[b]{4}{*}{ Pairs } & \multirow{4}{*}{$\begin{array}{l}\text { Eliminated } \\
\text { Pairs }\end{array}$} \\
\hline & 4 & $E,\{2\}$ & z & 5 & $G,\{2\}$ & & \\
\hline & Key & Value & z & Key & Value & & \\
\hline & 2 & $F, \varnothing$ & 흔 & 2 & $E, \varnothing$ & & \\
\hline \multirow{2}{*}{ Obj } & 5 & $F,\{2\}$ & $E$ & 2 & $F, \varnothing$ & \multirow{2}{*}{$\begin{array}{l}\text { E-F, E-G, } \\
F-G\end{array}$} & \multirow[t]{2}{*}{-} \\
\hline & 2 & $G, \varnothing$ & 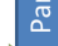 & 2 & $G, \varnothing$ & & \\
\hline G & 5 & $G,\{2\}$ & & 4 & $A,\{1\}$ & \multirow{5}{*}{$\begin{array}{l}\text { A-E, A-H, } \\
\text { A-I, B-E, } \\
\text { B-H, B-I, } \\
\text { E-H, E-I, }\end{array}$} & \multirow{5}{*}{$\begin{array}{l}\text { A-B: }\{1\} \cap\{1\} \\
H-I:\{3] \cap\{3]\end{array}$} \\
\hline H & 3 & $H, \varnothing$ & & 4 & $B,\{1\}$ & & \\
\hline \multirow[t]{3}{*}{1} & 4 & $H,\{3\}$ & & 4 & $E,\{2\}$ & & \\
\hline & 3 & $1, \varnothing$ & & 4 & $H,\{3\}$ & & \\
\hline & 4 & $I,\{3\}$ & & 4 & $I,\{3\}$ & & \\
\hline
\end{tabular}

Figure 12: Map Reduce phase after improvising the algorithm

Now the map phase emits key value pairs along with the keys smaller than the current key. For example, consider the entity A which has two common keys 1,4 where $\boldsymbol{\sigma}(\mathbf{A})=\{\mathbf{1}, \mathbf{4}\}$. The key value pair for the first time would be $(\mathbf{1},[\mathbf{A}, \varnothing])$, where $\varnothing$ represents that there are no keys smaller than the current key 1 . Now when the key 4 is considered, the map function gives $(\mathbf{4},[\mathbf{A},\{\mathbf{1}\}])$, which means that there is a key smaller than the current key 4. As $\boldsymbol{\sigma}(\mathbf{B})=\{\mathbf{1}, \mathbf{4}\}$ the pair A-B is compared with the key 1. Later when key 4 is considered then the $\boldsymbol{\sigma}_{\mathbf{4}}(\mathbf{A})=(\mathbf{4},[\mathbf{A},\{\mathbf{1}\}])$ has a subset of the key $\{1\}$.

The initial $(\mathrm{k}, \mathrm{v})$ i.e., $(\mathbf{1},[\mathbf{A}, \boldsymbol{\emptyset}])$ which is passed to the first reducer is compared against other entities who has common signature 1 . In this case it is B, C, D. So the pairs A-B, A-C, A-D, B-C, B-D, C-D are compared. Now the entities that share the signature 4 are sent to other reducer. As $\sigma(E)=\{2,4\}, \sigma(H)=\{3,4\}, \sigma(I)=\{3,4\}$ and now the pair A-B is ignored. In total, this process generated 26 pairs before the improvement of the algorithm. After the improvements made to the reducer it eliminated 4 pairs which is $15 \%$ less than the actual. So there is performance improvement. 
It is implemented in such a way that the entities with same keys fall under the same window and this window moves over rows of same obj and sorted by attributes. This approach uses two map jobs, one to emit key value pair for each key and another map job is used to group together the pairs having the same key.

\subsection{Real Time Example}

Consider that a dataset has following records

\begin{tabular}{|llll|}
\hline 1 & pari & sjsu & tirumali \\
2 & pari & JNTU & tiru \\
3 & pari & SJ & T \\
4 & pari & CA & Gandhi \\
5 & pari & CA & T.G \\
6 & pari & SJ & T.G \\
7 & pari & SJS & T \\
8 & pari & USA & tirumali \\
9 & pari & India & tirumali \\
10 & pari & Earth & tiru \\
11 & pari & pari & pari \\
12 & tiru & pari & tiru \\
\hline
\end{tabular}

Figure 13: Sample Table

And consider that there is one pass blocking and the map phase generates following key value pairs

\begin{tabular}{|lll|}
\hline pari, 1 & pari, 5 & pari, 9 \\
sjsu, 1 & CA, 5 & India, 9 \\
tirumali, 1 & T.G, 5 & tirumali, 9 \\
pari, 2 & pari, 6 & pari, 10 \\
JNTU, 2 & SJ, 6 & Earth, 10 \\
tiru, 2 & T.G, 6 & tiru, 10 \\
pari, 3 & pari, 7 & pari, 11 \\
SJ, 3 & SJS, 7 & pari, 11 \\
T, 3 & T, 7 & pari, 11 \\
pari, 4 & pari, 8 & tiru, 12 \\
CA, 4 & USA, 8 & pari, 12 \\
Gandhi, 4 & tirumali, 8 & tiru, 12 \\
\hline
\end{tabular}

Figure 14: Key value pairs generated by Map 


\begin{tabular}{|c|c|c|}
\hline pari, 1 & SJSU, 1 & Gandhi, 4 \\
\hline pari, 2 & tirumali, 1 & $C A, 4$ \\
\hline pari, 3 & tirumali, 8 & $\mathrm{Ca}, 5$ \\
\hline pari, 4 & tirumali, 9 & $T G$ \\
\hline parl, S & JNTU, 2 & T.G, 6 \\
\hline 7 & tiru, 2 & SJS, 7 \\
\hline pari, 8 & tiru, 10 & USA. 8 \\
\hline pari, 9 & tiru, 12 & India, 9 \\
\hline pari, 10 & tiru, 12 & earth, 10 \\
\hline pari, 11 & $\mathrm{SJ}, 3$ & \\
\hline pari, 11 & $\mathrm{SJ}, 6$ & \\
\hline pari, 11 & $\mathrm{~T}, 3$ & \\
\hline pari, 12 & $\mathrm{~T}, 7$ & \\
\hline
\end{tabular}

Figure 15: Grouping similar entities

pari, $<1,2,3,4,5,6,7,8,9,10,11,12>$
SJSU $<1>$
tirumali $\langle 1,8,9>$
JNTU $<2>$
tiru $<2,10,12>$
SJ $<3,6>$
T $<3,7>$
CA $<4,5>$
Gandhi $<4>$
T.G $<5,6>$
SJS $<7>$
USA $<8>$
India $<9>$
earth $<10>$

Figure 16: Sorting the keys associated with each entity 
The reducer creates following entity pairs

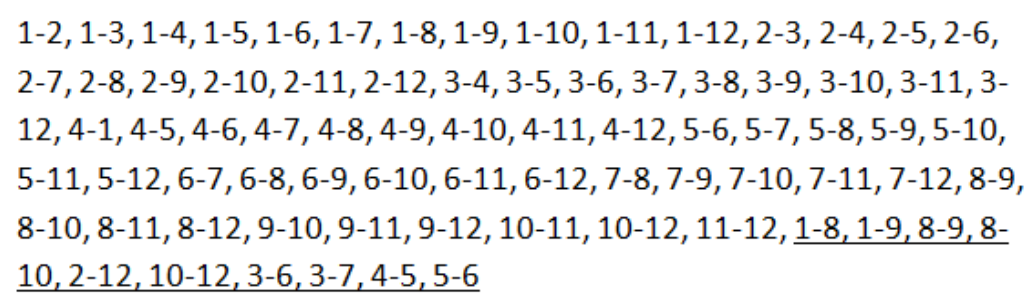

Figure 17: Entity pairs

Total number of pairs $=76$

Redundant pairs $=10$

Efficiency improvement $=(10 / 76) * 100=13.15 \%$ 


\section{Implementation Details}

This section describes about the algorithm implemented as part of baseline, the dataset chosen for experimentation and other tools and technologies used throughout the implementation of this project are explained.

The problem definition section has clearly described about the solution for how to avoid the redundant comparison. In this section I will describe about how I used Sparks libraries to solve this problem.

\subsection{Baseline Implementation 1}

In the paper referred the author talks about using the index of the string valued key instead of using the string itself. So as a part of the initial implementation, I implemented the algorithm using this technique. I created index called ID which is a unique value for every record present in the dataset and created a indexed file and the data is saved in the following format. For this implementation I used Medical Health contacts dataset and it had some 38 columns and most of them has null values or most of the columns had the same values for almost all the records. Using such data will not be of much help for this implementation. So I chose only few columns which are mostly not null. I chose Agency name, phone number, toll free number, email and web address columns.

\section{[ID,[AGENCY, LOCALPHONE, TOLLFREEPHONE, EMAIL, WEB]]}

The indexed file is generated as shown in the following figure and each spark partition can handle an RDD of $2 \mathrm{~GB}$. With this extraordinary feature of spark, we can give large amounts of data to process and spark does it very easily. 


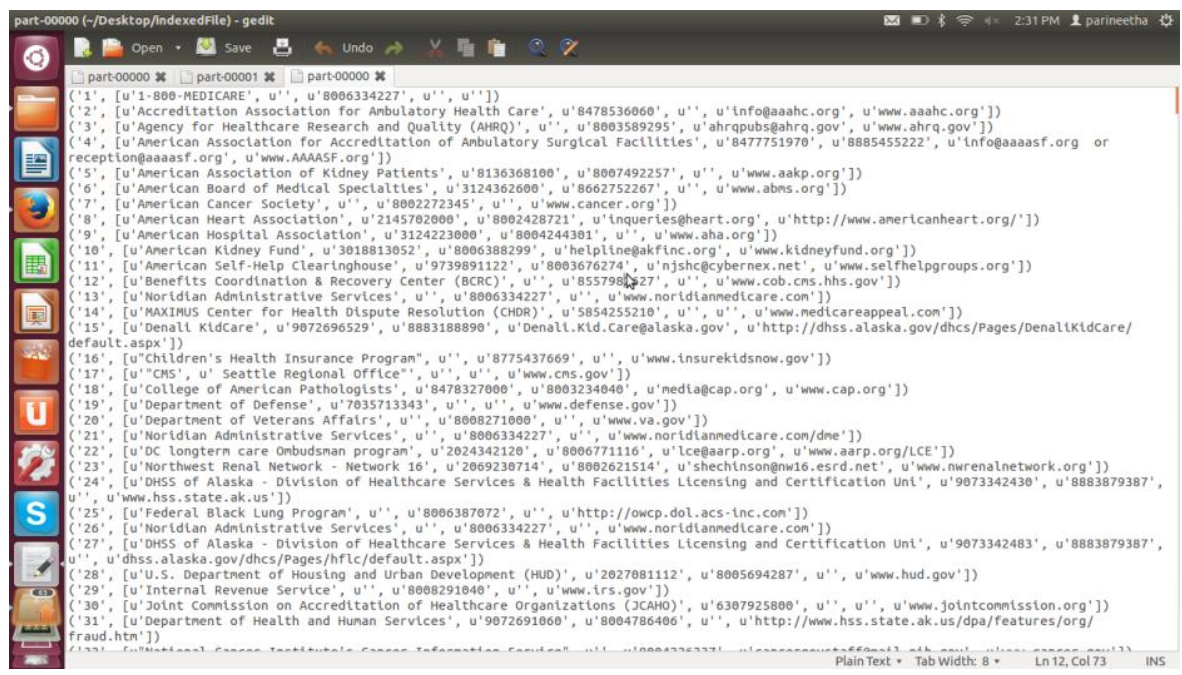

Figure 18: Snapshot of indexed file

When there are multiple values in a row, Spark considers the first value as the key and all other values as values. As the first value(key) in the indexed file is ID, map function is used to swap the value with the key and make value as key. This was just an idea given by the author, implemented it to test for the match entities within a file and it worked. Following line of code is used to get the key value pairs as we needed

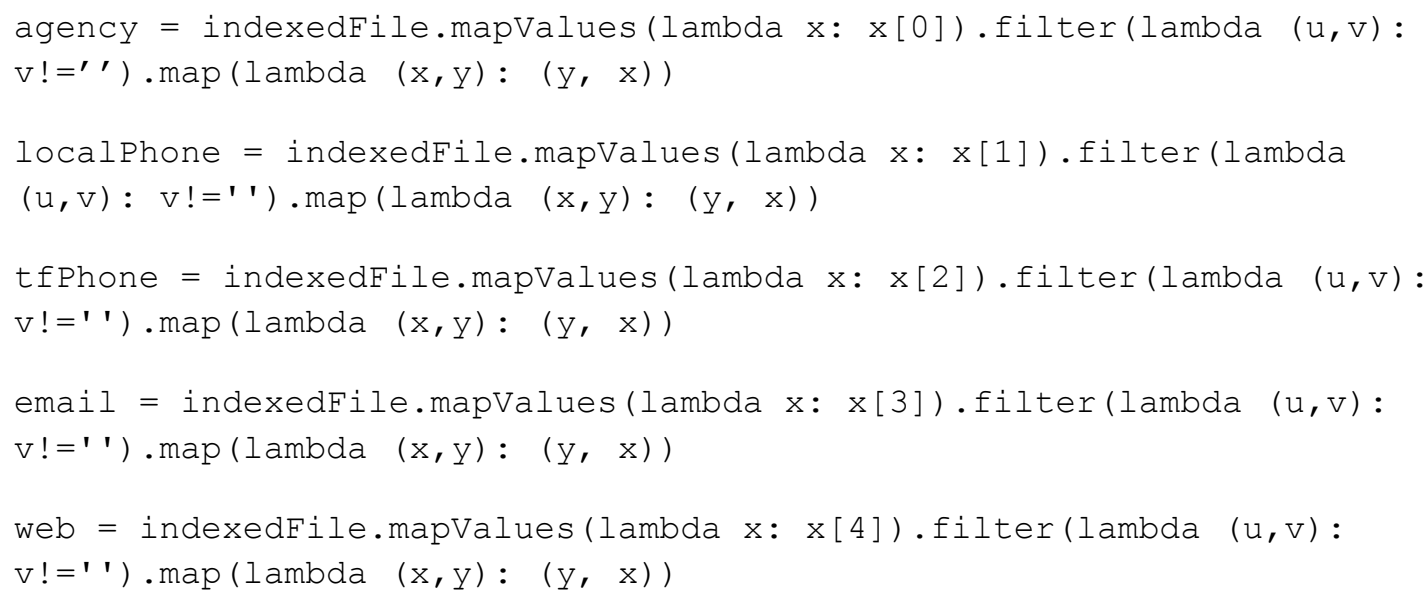

Figure 19: Code snippet to generate key value pairs from map 
Same filter operation is performed on all other keys such as LOCALPHONE, EMAIL, TOLLFREEPHONE, WEB.

And I generated the file as shown in the following table

\begin{tabular}{|l|}
\hline [KEY, VALUE] \\
\hline [AGENCY, Row_ID] \\
\hline [LOCALPHONE, Row_ID] \\
\hline [TOLLFREEPHONE, Row_ID] \\
\hline [EMAIL, Row_ID] \\
\hline [WEB, Row_ID] \\
\hline
\end{tabular}

Figure 20: Snapshot of indexed file contents

Later entities having the same keys are grouped together and a list is generated to group entities having the same key

Using the groupbykey operation provided by spark on [AGENCY, Row_ID] and other attributes, I created a new dataset consisting of [AGENCY, Iterable $<$ Row_ID $>$ ] and other attributes with their respective iterable value. After grouping all the attributes I sorted their values in ascending order using the following line of code.

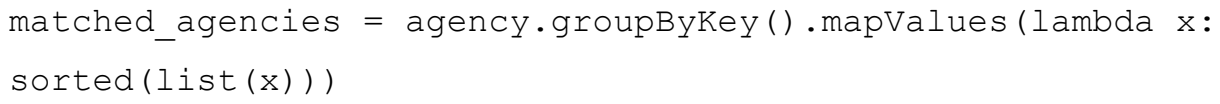

[KEY, SORTEDSIGNATURES]
[AGENCY, SORTED(LIST<ID>)]
[LOCALPHONE, SORTED(LIST $<\mid \mathrm{DD}>$ )]
[TOLLFREEPHONE, SORTED(LIST $<$ ID>)]
[EMAIL, SORTED(LIST<ID>)]
[WEB, SORTED(LIST<ID>)]

Figure 21: Snapshot of Entity, SortedSignatures

Generating entity pairs: To generate pairs, first element is taken and paired with rest of the elements until there's no element left in list. Following function is used to generate pair of IDs out of list of IDs 


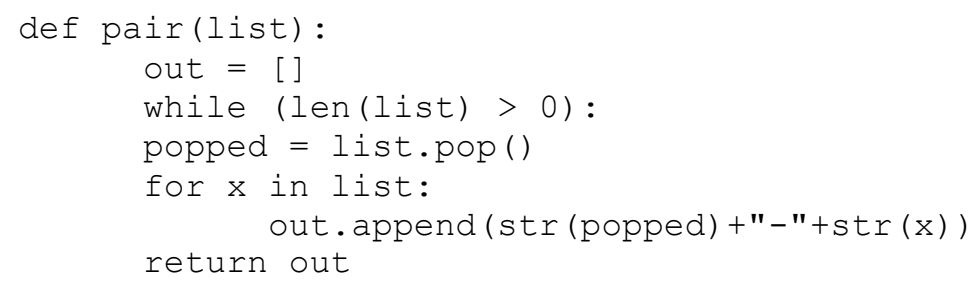

Figure 22: Code snippet for generating entity pairs

Using above defined pair function to work on each sorted list of IDs. flatMapValues() generates the pairs of IDs out of list of IDs, and rather than returning list of pairs, puts each pair in new line.

flat_matched_agencies = matched_agencies.flatMapValues(lambda x: pair (x))

[Key, PairOfEntities]
[AGENCY, ID_Pair]
[LOCALPHONE, ID_Pair]
[TOLLFREEPHONE, ID_Pair]
[EMAIL, ID_Pair]
[WEB, ID Pair]

Figure 23: Snapshot of Key value pairs in the form of key and pairs of entities

This was a trial implementation of first baseline. The output of it is shown in the following figure.

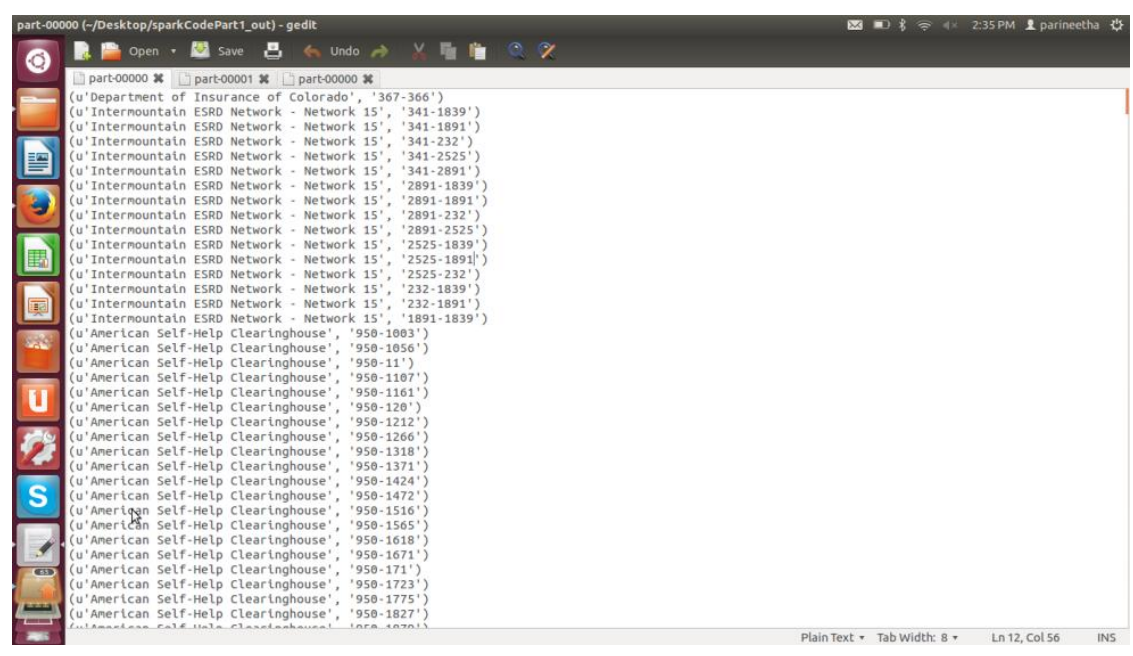

Figure 24: Generation of Entity Pairs 


\subsection{Baseline Implementation 2}

When implementing the algorithm on big data, it is very important to check the quality of the data. In the dataset I chose there were many data quality issues like

New line characters: When I ran the code I was not aware of the new line characters present in few records. Debugging and figuring out what the problem was very time consuming. There were very few attributes which had new line character, so to retain the consistency these records were deleted.

Null values: As the dataset is very large it is expected to have null values. There were many fields in the dataset which had $90 \%$ of the records as nulls. Considering such fields would just create lag in the execution time with no positive effect on the result. So these fields were removed.

Baseline implementation 2 is more Spark oriented. The main goal of this paper is to implement efficient pair-wise similarity computation using Spark. To implement the algorithm suggested in the paper I used the concept of Data Frames and Windows from the Spark libraries.

Input: Medical Health dataset with 996,000 records.

Output: Output generated two files named eliminated and matched. Matched file has all the pair of entities that matched only once. Eliminated file has all the pairs of records that were supposed to be matched redundantly.

Made use of following pyspark libraries

pyspark.sql.Window: A distributed collection of data grouped into named columns. pyspark.sql.Row: A row of data in a DataFrame.

pyspark.sql.HiveContext: Main entry point for accessing data stored in Apache Hive

4.2.1 Hive Context: It is a superset of SQL Context as it provides all the functionalities of SQL and Hive as well. 
4.2.2 Data Frames: Data frames in spark provide the flexibility of collecting the data from various data sources such as resilient distributed dataset or external files or Hive tables, etc. In simple terms it is like creating a table with named attributes. To create a data frame we first created the schema, column names and their datatypes.

- Generate a new RDD out of list/ tuple of already existing RDDs

aoPair = inRDD.flatMap(lambda line: attr_key(line.split("|t")))

- Define the schema for the data frame. Spark has become so advanced that if the datatype of the column is not mentioned then it will try to infer from the data what type it can be by going through some amount of the data which is called sampling ration[11].

\section{schema $=$ StructType([StructField("attr", StringType(), True), StructField("obj", StringType(), True)])}

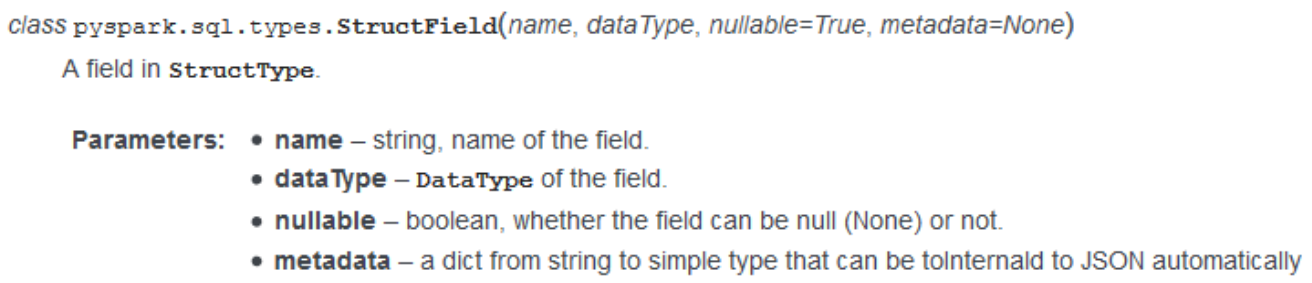

- Finally using the createDataFrame function apply the above schema to the RDD aoDF = sqlCtx.createDataFrame (aoPair, schema $)$ 


\begin{tabular}{|c|c|}
\hline attr & obj \\
\hline 1 & $a$ \\
\hline 2 & $a$ \\
\hline 3 & $a$ \\
\hline 1 & $b$ \\
\hline 2 & $b$ \\
\hline 3 & $b$ \\
\hline 1 & $c$ \\
\hline 3 & $c$ \\
\hline
\end{tabular}

Figure 25: List of attributes and objects

\subsubsection{Window Concept in Spark}

Once the data frame is created with the mentioned schema, we need to perform operations on this data frame. I used the concept called Windows in Spark. Apache Spark allow us to perform certain functionalities on group of rows. These group of rows is known as window. By defining a window we can perform some operations on data frames[11]. Spark SQL provides 3 kinds of aggregate functions on windows: Ranking, Analytic and Aggregate functions. window object is in the package called pyspark.sql, So we need to import it using the following line of code

\section{from pyspark.sql import HiveContext, Row, Window}

$$
\text { memorize = aoDF.select("attr", "obj", lag("attr",1, None).over(window).alias("prev")) }
$$

The lag method gets the previous records for the current record and the parameter 1 represents get it from the one previous row and the parameter None represents what to do when there is no previous value. over(window) defines a windowing column and to jumps over one window at a time. If the current window is for the obj "a" then it process the window 'a' and then only jumps to the other window and process that. The alias function returns a new data frame and in this case we are creating a new data frame called "prev". 


\begin{tabular}{|c|c|c|}
\hline attr & obj & prev \\
\hline 1 & a & null \\
\hline 2 & $a$ & 1 \\
\hline 3 & $a$ & 2 \\
\hline 1 & $b$ & null \\
\hline 2 & $b$ & 1 \\
\hline 3 & $b$ & 2 \\
\hline 1 & c & null \\
\hline 3 & c & 1 \\
\hline
\end{tabular}

Figure 26: List of attributes, objects and previous values

Finally the data frame created looks like

DataFrame : [attr, obj, prev] $\rightarrow$ RDD : [(attr, (obj, prev))]

\begin{tabular}{|c|c|}
\hline attr & (obj, prev) \\
\hline 1 & (u'a', None) \\
\hline 2 & $\left(u^{\prime} a^{\prime}, 1\right)$ \\
\hline 3 & $\left(u^{\prime} a^{\prime}, 2\right)$ \\
\hline 1 & (u'b', None) \\
\hline 2 & $\left(u^{\prime} b^{\prime}, 1\right)$ \\
\hline 3 & $\left(u^{\prime} b^{\prime}, 2\right)$ \\
\hline 1 & (u'c', None) \\
\hline 3 & (u'c', 1) \\
\hline
\end{tabular}

Figure 27: List of attributes and object, previous pairs

mappedRDD = memorize $\cdot$ map $($ lambda row: $($ row $\cdot$ attr, $($ row.obj, row.prev $)))$

groupedByAttr $=$ mappedRDD.groupByKey().mapValues(list).cache()

[(1, [(u'a', None), (u'b', None), (u'c', None)]), (2, [(u'a', 1), (u'b', 1)]), (3, [(u'a', 2), (u'b', 2), $\left.\left.\left.\left(u^{\prime} c^{\prime}, 1\right)\right]\right)\right]$

Later grouped it by attribute and collected the tuple (obj, prev) into the list 
Implemented following algorithms by making use of the spark libraries

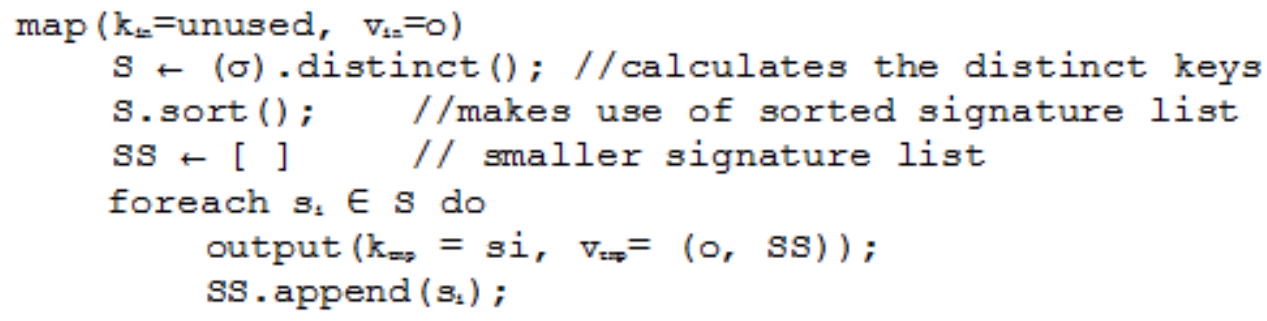

Figure 28: Algorithm for Map Phase

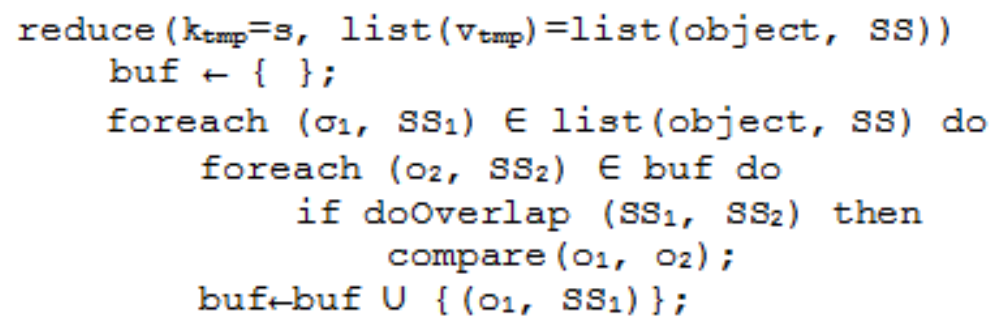

Figure 29:Algorithm for Reduce Phase

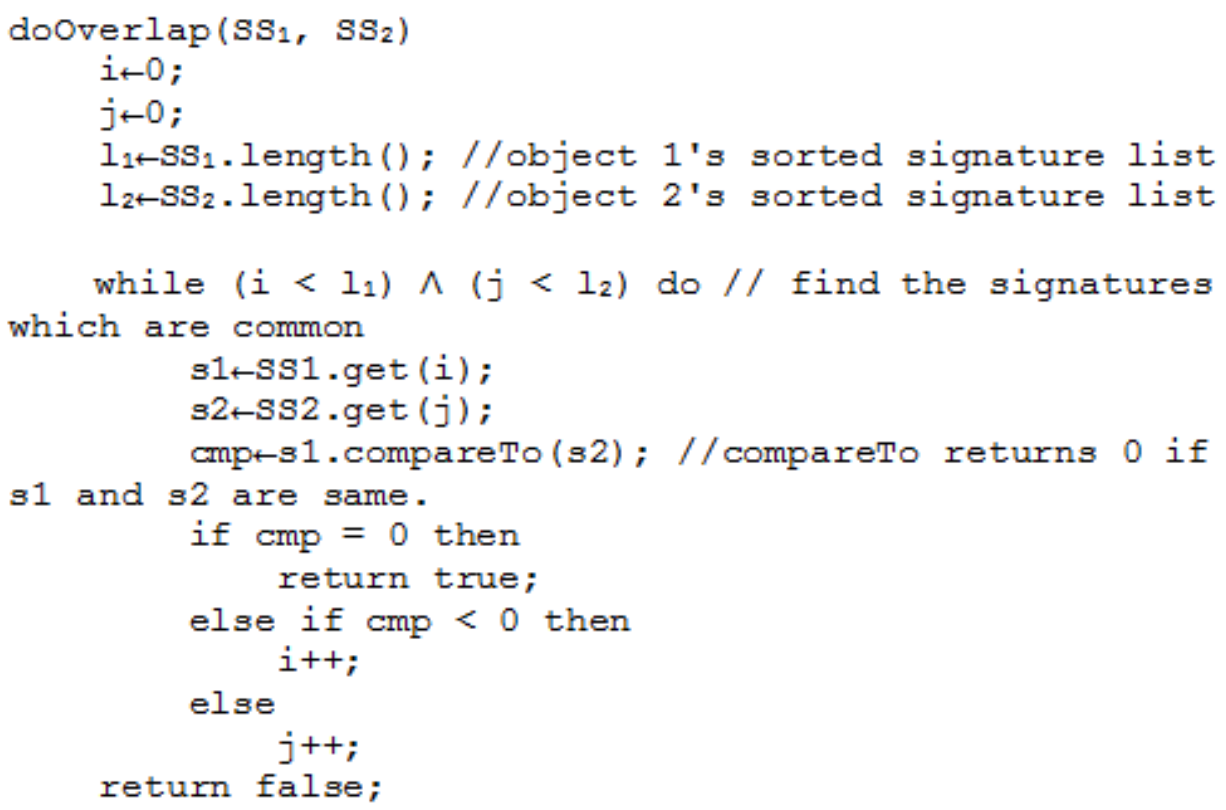

Figure 30:Algorithm for Overlap 


\section{Performance Evaluation}

I have performed the implementation on Spark cluster on virtual machines. This set up had 3 nodes with one as master node and two others as slave nodes. Each node is of 2GB RAM and installed with Ubuntu 14.04. Experimentation is done using Apache Spark version 1.4 with 2.4 Hadoop distribution. The data frames concept in spark was introduced in the version 1.3, so I had to use version 1.3 or beyond for this implementation.

Tested the implementation in the Spark standalone cluster mode on Medical Health dataset called Plaid which had 996000 records. Without the implementation of this algorithm the simple Cartesian product have done matching in very naive, compare one record with each other record present in the dataset. With this implementation we have narrowed down the search space and reduced the comparisons 163,527. We get 119,369 records as matching and eliminated 44158 matches which reduced the number of comparisons by $44158 / 163527=27 \%$ of the records are eliminated as redundant.

Execution time with and without redundant pairs

\begin{tabular}{|c|c|c|}
\hline $\begin{array}{l}\text { Number } \\
\text { of records }\end{array}$ & $\begin{array}{l}\text { Execution time with redundant } \\
\text { pairs(minutes) }\end{array}$ & $\begin{array}{l}\text { Execution time without } \\
\text { redundant pairs(minutes) }\end{array}$ \\
\hline $100 \mathrm{k}$ & 34.1 & 31.7 \\
\hline $300 k$ & 68.8 & 65 \\
\hline 500k & 166.6 & 105.4 \\
\hline $700 \mathrm{k}$ & 158 & 149.8 \\
\hline 996k & 225.8 & 213.6 \\
\hline
\end{tabular}

Table 1: Execution time with and without redundant pairs 


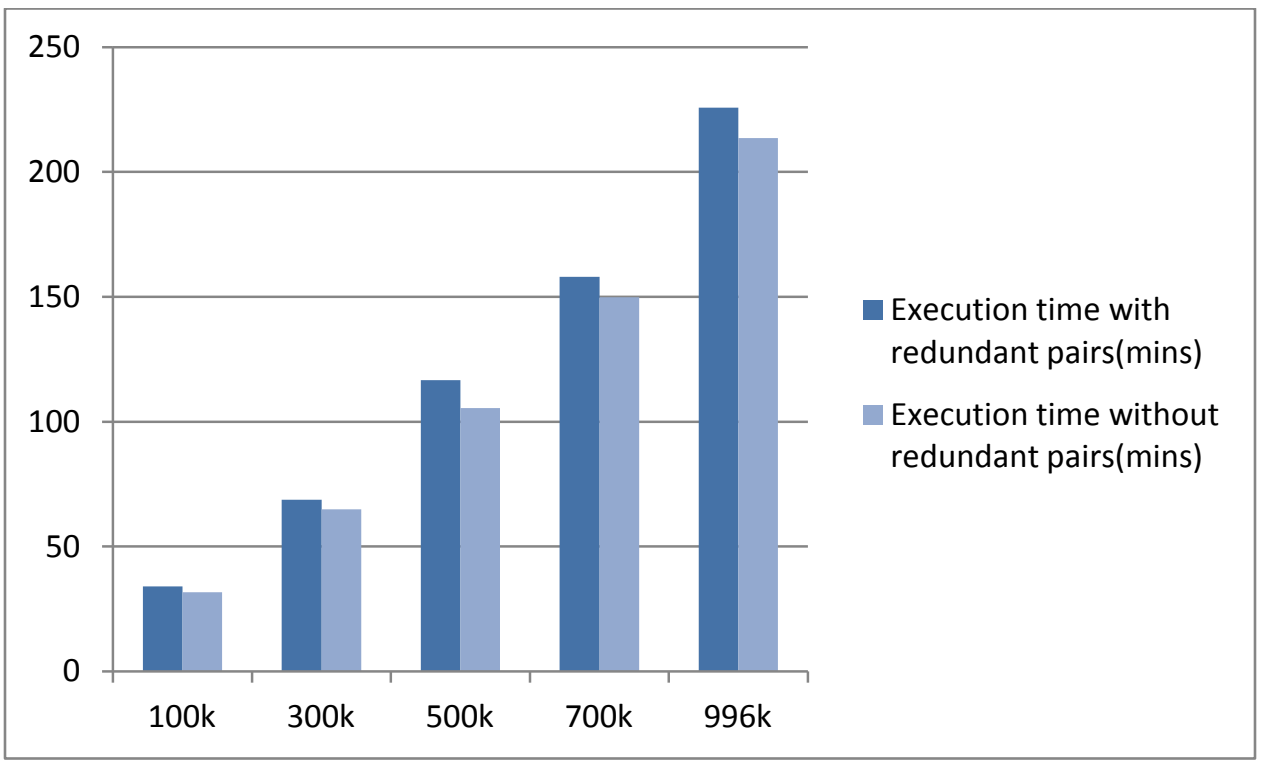

Figure 31: Execution times comparison

\subsection{Apache Spark Cluster Setup}

Implementation is done in Virtual Machine mode where using VM Workstation and created two virtual machines with Ubuntu version 1.4 installed. Configured these VMs with 2 GB RAM, 20GB hard disk and quad core processor.

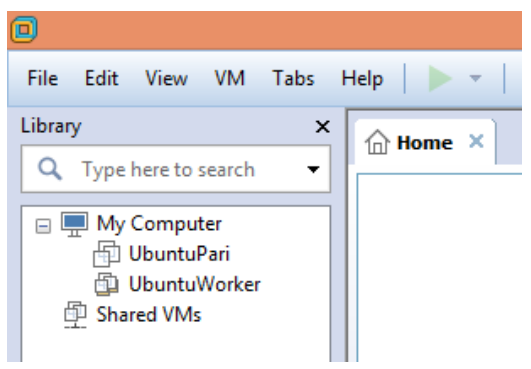

Figure 32: Screenshot of two VMs in VMWare WorkStation

Install Java on the VM and update the JAVA_HOME in environmental variables

JAVA_HOME=/usr/lib/jvm/java-6-oracle/ 


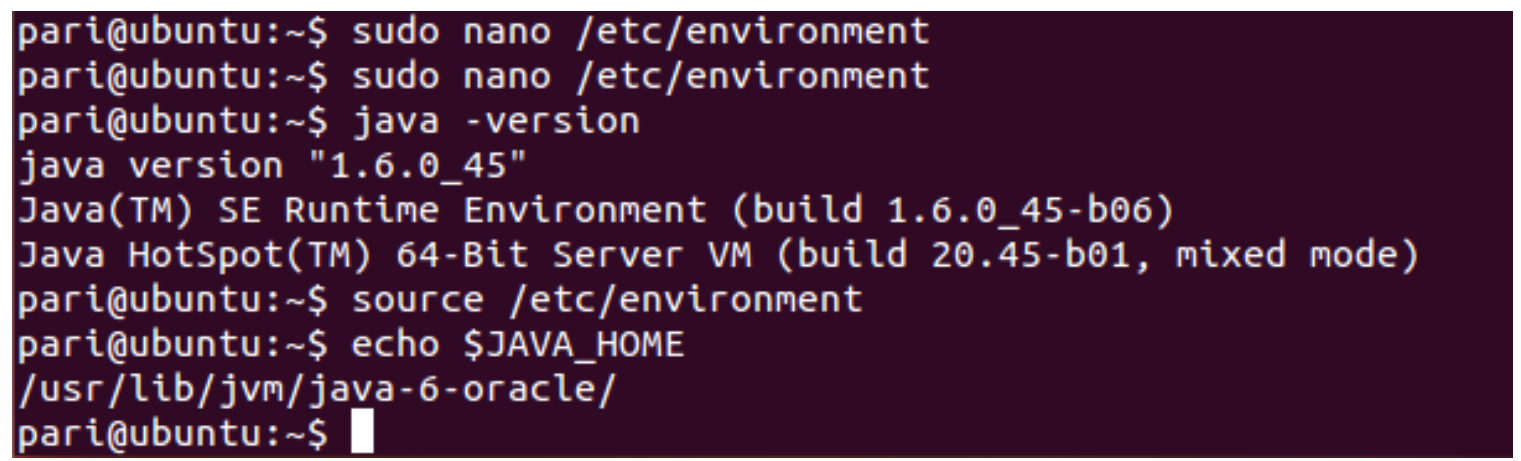

Figure 33: Screenshot showing Java version on VM

Download debian version of Scala if you are using Ubuntu

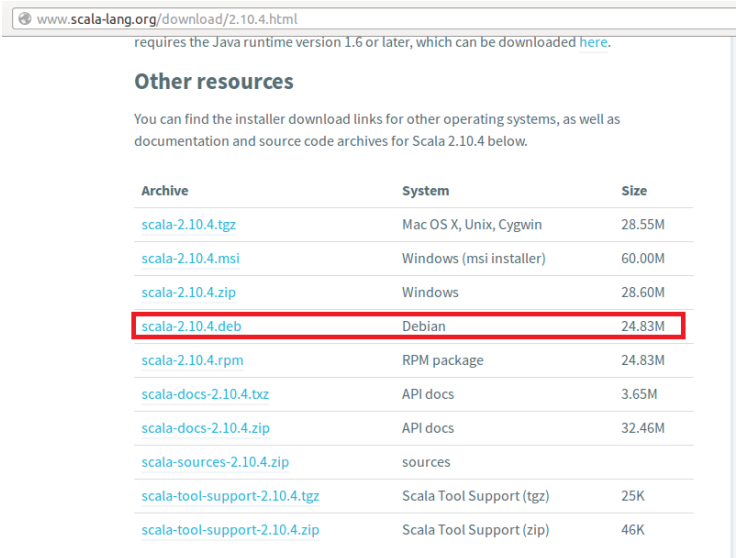

Figure 34: Screenshot for Scala download

and install it from the software center. I installed 2.10.4 version

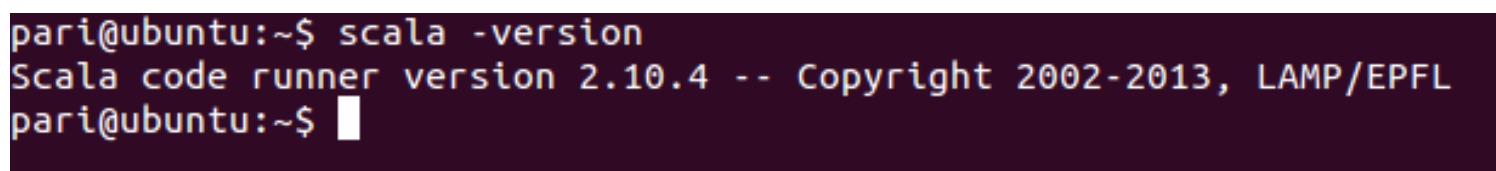

Figure 35: Screenshot showing Scala version on VM

Clone the master node to get the virtual machine with all the softwares installed till now. With few modification we set the second virtual machine as Worker node. 


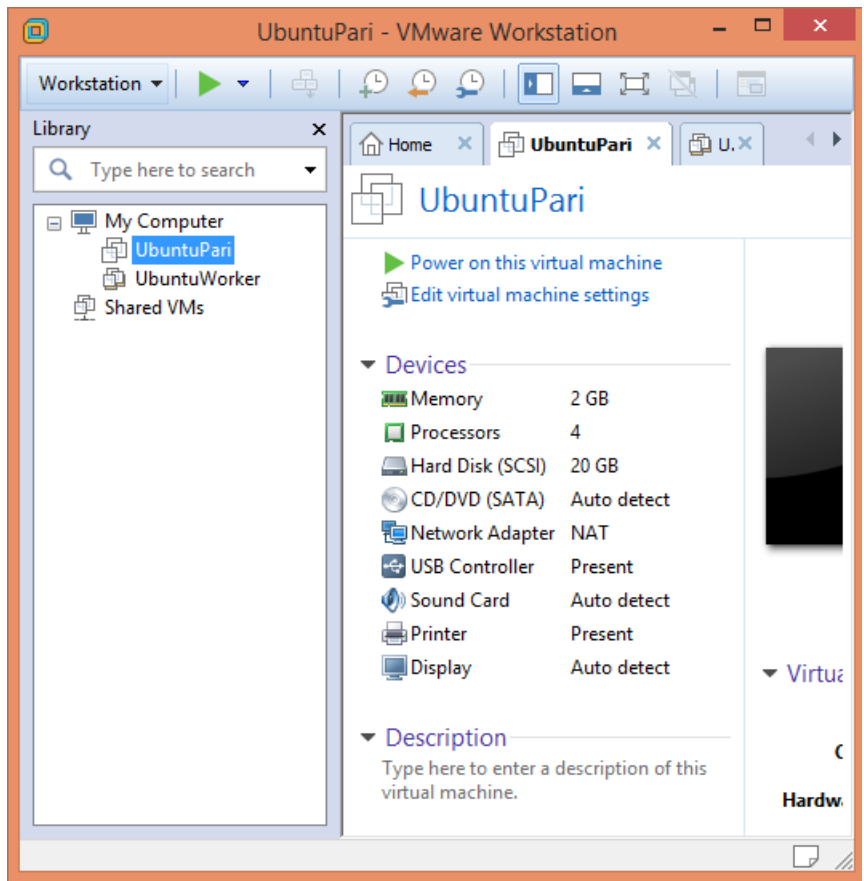

Figure 36: Screenshot of Master Node

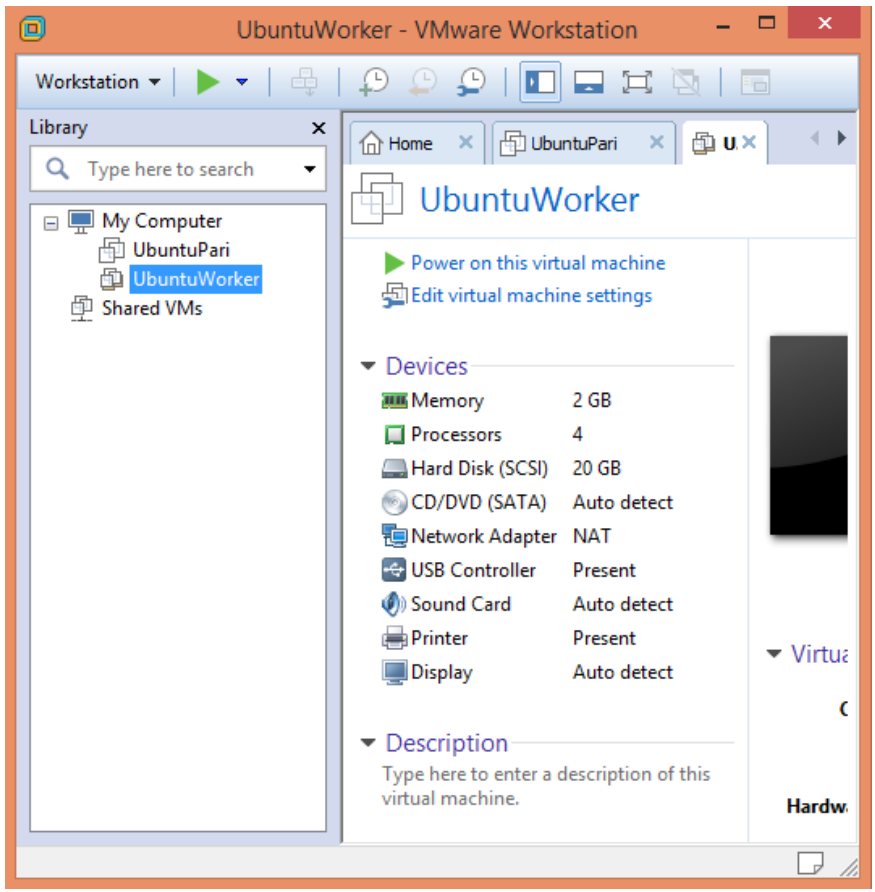

Figure 37: Screenshot of Worker Node 
Once both machines are ready, install ssh on worker node to give access to the master node to access worker.

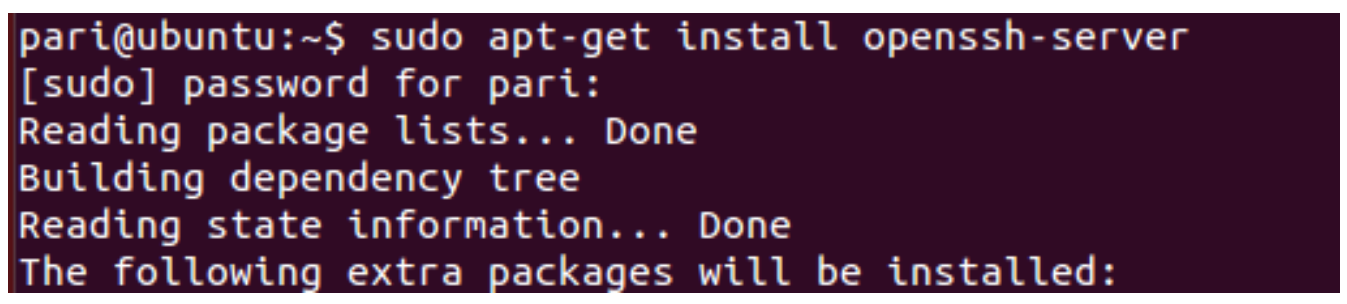

Figure 38: Screenshot for installing ssh

Later RSA key needs to be generated on the master node to obtain remote access. Connect the master node with the worker node. Copy the public key generated earlier to each worker node. This gives master to access worker node with SSH

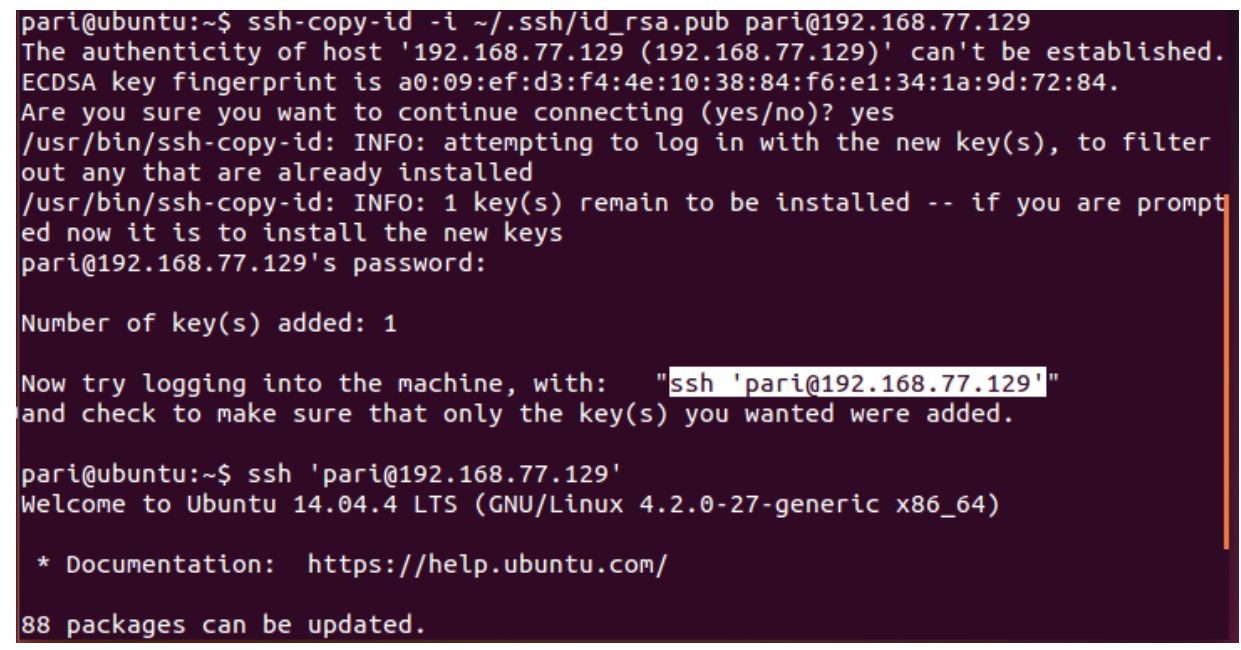

Figure 39: Screenshot showing Master connection

Create a new VM and repeat the same process and take screenshots

Download and install required version of spark on both the nodes. 


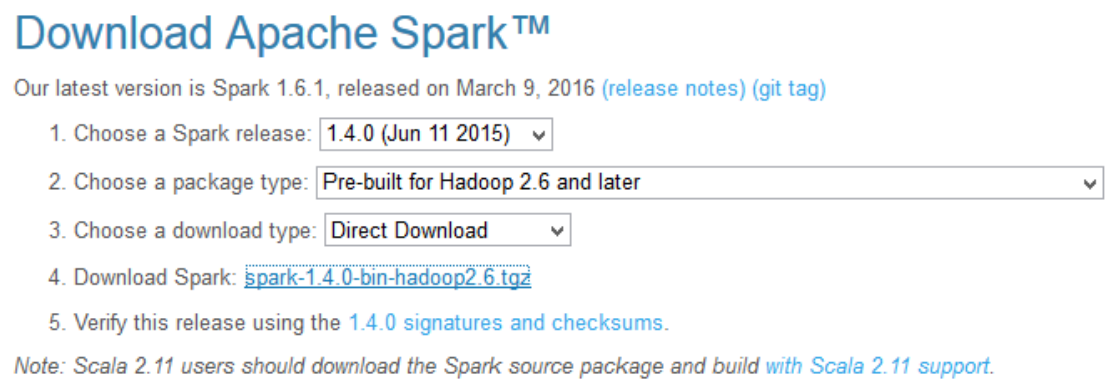

Figure 40: Spark version

Until now these two nodes are the same as we have not given master or worker specifications to any node. The main settings should be made in the conf file of the spark package. conf file consists of following files

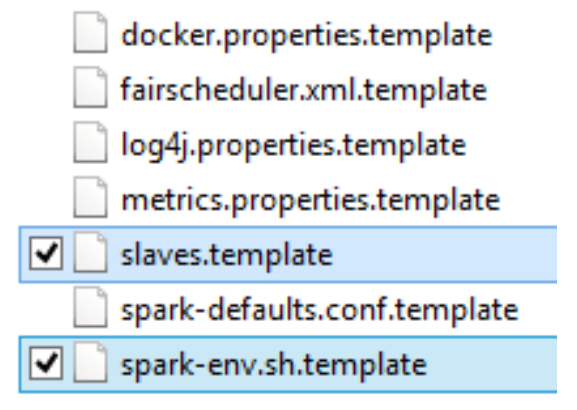

Figure 41: Files in conf folder

Make a copy of slaves.template file and spark-env.sh.template file.

Rename "slaves.template(copy)" to "slaves" and "spark-env.sh.template(copy)" to "sparkenv.sh".

Add the IP address of the worker nodes in the slaves file. 


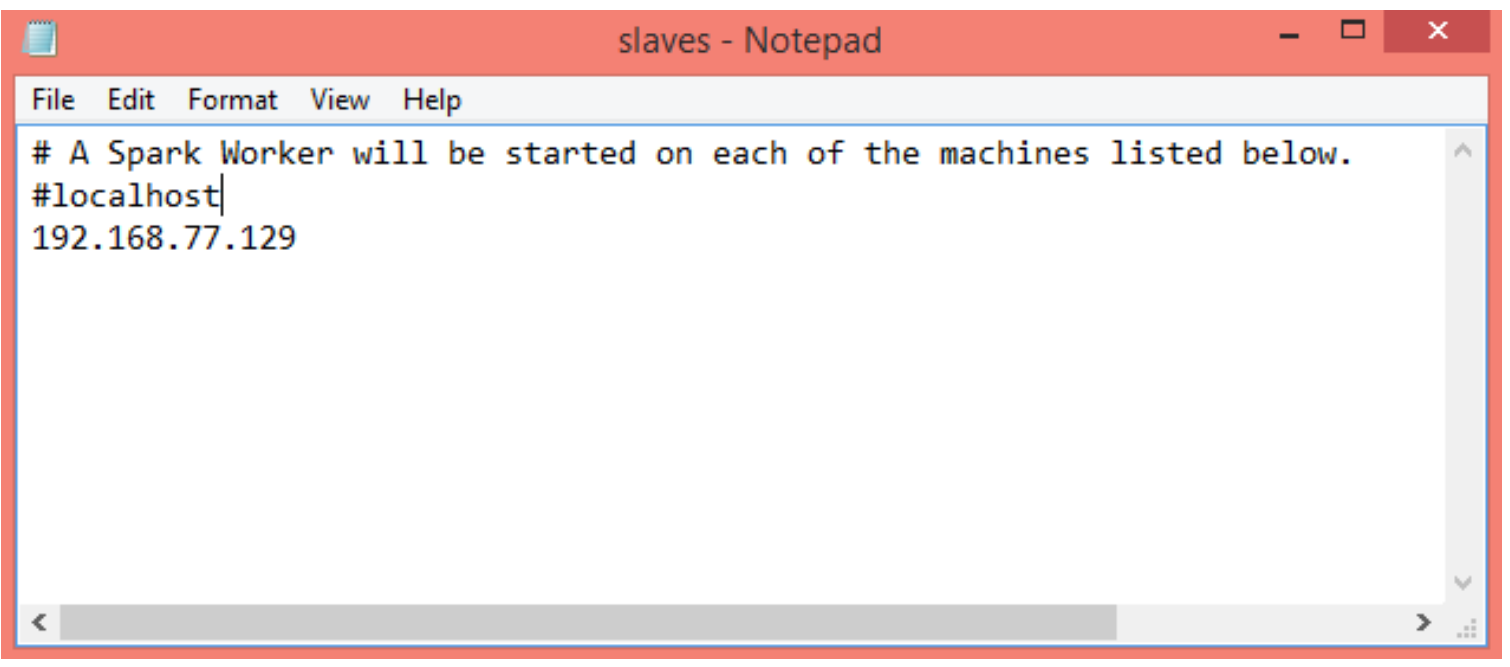

Figure 42: Updated Slaves file

Add the following lines in spark-env.sh file.

export SPARK_MASTER_IP=192.168.77.130

export SPARK_WORKER_CORES=1

export SPARK_WORKER_MEMORY=800m

export SPARK_WORKER_INSTANCES=2

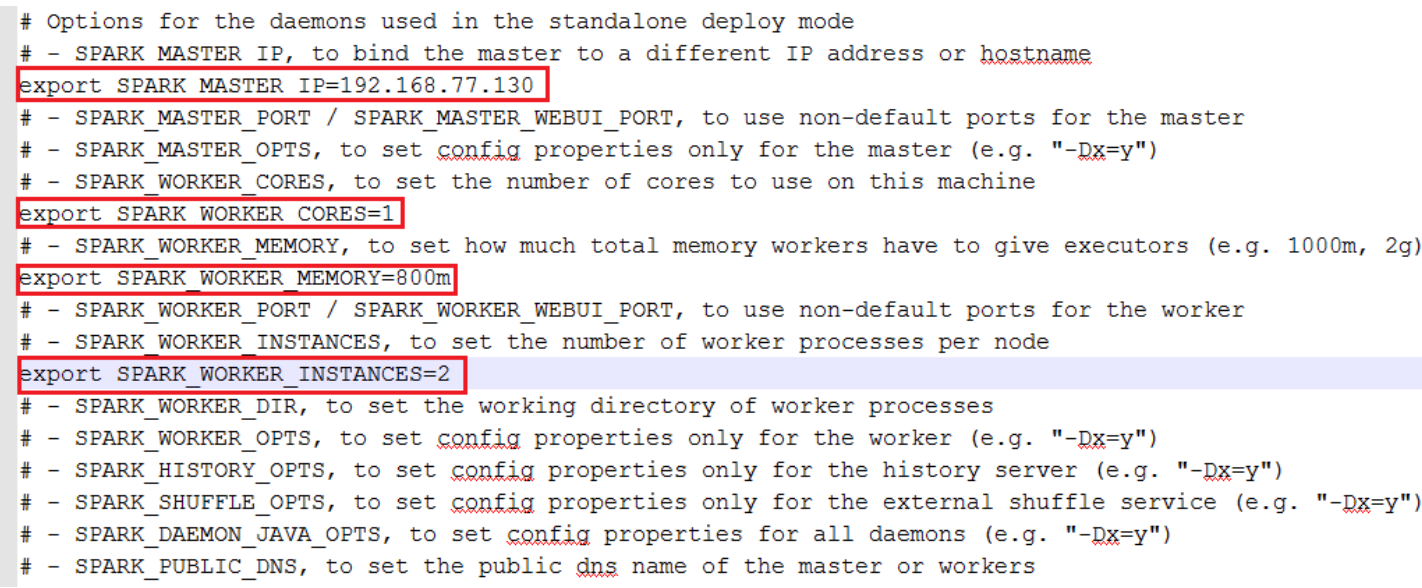

Figure 43: Updated spark-env.sh file

To test the installation, run 
./bin/spark-shell

scala:> sc.parallelize(1 to 1000).count();

(This should give the output as long=1000)

scala:>exit

.bin/run-example SparkPi

(This should give the output of calculated pi value)

\subsection{Launching and testing the cluster}

The sbin folder has the files to start or stop a master or slave

To start the cluster we need to execute following command in the terminal

./sbin/start-all.sh

and to stop

./sbin/stop-all.sh 


\begin{tabular}{l}
$\square$ Name \\
$\square$ slaves.sh \\
$\square$ spark-config.sh \\
$\square$ spark-daemon.sh \\
$\square$ spark-daemons.sh \\
\hline$\square \square$ start-all.sh \\
\hline$\square$ start-history-server.sh \\
\hline$\square$ start-master.sh \\
$\square$ start-mesos-dispatcher.sh \\
$\square$ start-shuffle-service.sh \\
$\square$ start-slave.sh \\
$\square$ start-slaves.sh \\
$\square$ start-thriftserver.sh \\
$\square \square$ stop-all.sh \\
\hline$\square$ stop-history-server.sh \\
\hline$\square$ stop-master.sh \\
$\square$ stop-mesos-dispatcher.sh \\
$\square$ stop-shuffle-service.sh \\
$\square$ stop-slave.sh \\
$\square$ stop-slaves.sh \\
$\square$ stop-thriftserver.sh
\end{tabular}

Figure 44: Files in sbin folder 


\section{Conclusion}

This project implements pair-wise similarity computation without redundancy using Apache Spark. The implementation is done successfully on large dataset consisting of 996000 records. As Apache spark is a powerful big data processing engine which has the concept of windows and data frames using which we tried improving this area of entity matching has scope for more improvement. Improving the concept of redundant comparison is a very rare study. This project can be further improvised by implementing multiple blocking strategies. It can be implemented using Amazons cluster and try it for more larger dataset. This algorithm with further research and slight modifications can also be tested upon the datasets consisting of images, text and videos. 


\section{References}

[1] Hanna Köpcke, Erhard Rahm, Frameworks for Entity Matching: A comparison. Database Group, University of Leipzig, Postfach 100920, 04009 Leipzig, Germany, Journal Data \& Knowledge Engineering, volume 69, issue 2, pages 197-210, 2009 http://dl.acm.org/citation.cfm?id=1672420

[2] George Papadakis, Ekaterini Ioannou, Claudia Niederée, Themis Palpanas, and Wolfgang Nejdl: Eliminating the Redundancy in Blocking-based Entity Resolution Methods, In Proceedings of the 11th annual international ACM/IEEE joint conference $\begin{array}{lllll}\text { on Digital } & \text { libraries, } & \text { pages } & 85-94, & \end{array}$ http://dl.acm.org/citation.cfm?id=1998093\&dl=ACM\&coll=DL\&CFID=619571752 $\&$ CFTOKEN $=36873886$

[3] Lars Kolb, Andreas Thor, Erhard Rahm: Don't Match Twice: Redundancy-free Similarity Computation with MapReduce, In Proceedings of the Second Workshop on Data Analytics in the Cloud, pages 1-5, 2013 http://dl.acm.org/citation.cfm?id=2486768

[4] Jimmy Lin: Brute force and indexed approaches to pairwise document similarity comparisons with MapReduce, Proceedings of the 32nd international ACM SIGIR conference on Research and development in information retrieval, pages 155-162, 2009 http://dl.acm.org/citation.cfm?id=1571970

[5] Groß, A., Hartung, M., Kirsten, T., Kolb, L., Köpcke, H., \& Rahm, E. Data Partitioning for Parallel Entity Matching. CoRR, abs/1006.5309, 2010.

[6] Lars Kolb, Erhard Rahm: Parallel Entity Resolution with Dedoop, volume 13, issue 1, pages 23-32, Journal Datenbank- Spektrum, 2013, http://link.springer.com/article/10.1007/s13222-012-0110-x

[7] [Online, accessed on January 2015] Apache Spark programming guide: http://spark.apache.org/docs/latest/programming-guide.html

[8] [Online, accessed on August 2015] Installing Apache spark on standalone cluster mode: http://www.trongkhoanguyen.com/2014/11/how-to-install-apache-spark-121in.html 
[9] [Online, accessed on August 2015] PySpark Guide: https://cwiki.apache.org/confluence/display/SPARK/PySpark+Internals

[10] [Online, accessed on August 2015] pyspark.sql.DataFrame and window Guide: http://spark.apache.org/docs/latest/api/python/pyspark.sql.html\#pyspark.sql.DataFram $\mathrm{e}$

Image References:

1. [Online, accessed on February 2016] http://studybigdata.co/data-flow/

2. [Online, accessed on February 2016] http://docplayer.net/6327218-Hadoopmapreduce-and-spark-giorgio-pedrazzi-cineca-scai-school-of-data-analytics-andvisualisation-milan-10-06-2015.html 